\title{
FILTROS ÓTIMOS: EFEITOS DE JANELAS E DE TIPO DE FASE
}

\author{
Lourenildo W. B. Leite', Fábio J. C. Alves² \\ Submetido em 6 maio, 2003/Aceito 18 março, 2004 \\ Submited May 6, 2003/Accepted March 18, 2004
}

\section{ABSTRACT}

The central objective under investigation in this paper is the performance of WHL deconvolution operators for the compression of the seismic source-pulse under the conditions of non-minimum phase and density of events on the trace, as is the case supported for the real data and batch deconvolution. The method of attack to the constructed problems is centered on the information content of the autocorrelation function submitted to different conditions of: (a) the truncation and taper windows; (b) the characteristics of the operator phase (if minimum or non-minimum phase); $(c)$ the measure of quality; (d) the whitening level; (e) the noise present and equalization; $(\mathrm{f})$ the balancing of the trace; $(\mathrm{g}$ ) the physical principles of wave propagation as expressed and limited by the convolutional model. Results can only be obtained numerically, and they are shown in the form of albums with increasing difficulties, and they demonstrate how the time windows on the autocorrelation serve to diagnostic and to improve the performance of the operators. We conclude that many questions still arise when deconvolution techniques are applied to seismic reflection data from sedimentary basins, and that the Goupillaud model is convenient for simulations through its simple and complete mathematical description.

Keywords: Deconvolution, Wiener-Hopf-Levinson (WHL), Goupillaud, Minimum phase.

\section{RESUMO}

0 objetivo central deste trabalho é o estudo do desempenho do operador deconvolucional WHL na compressão do pulso-fonte sísmico, sob condições especiais de fase não-mínima e da densidade de eventos no traço, como casos advogados para dados reais e processamento em rotina. 0 método de ataque ao problema construído é centrado no conteúdo da informação da função autocorrelação submetida a diferentes condições: (a) de truncamento e tipo de janelas; (b) das características da fase do operador (se mínima ou não-mínima); (c) da medida de qualidade; (d) do nível de embranquecimento; (e) do ruído presente e da equalização; $(f)$ do balanceamento do traço; $(g)$ dos princípios físicos da propagação expressos e limitados pelo modelo convolutional. Os resultados obtidos são apenas na forma numérica, organizados na forma de álbuns com dificuldades crescentes, e demonstram como 0 uso de janelas na autocorrelação serve para diagnosticar e melhorar a performance dos operadores. Concluímos que muitas perguntas ainda surgem quando técnicas de deconvolução são aplicadas a seções sísmicas de bacias sedimentares, e que o modelo de Goupillaud é conveniente para simulações e análises devido a sua descrição matemática simples e completa.

Palavras-chave. Deconvolução, Wiener-Hopf-Levinson (WHL), Goupillaud, fase mínima.

\footnotetext{
Universidade Federal do Pará. Centro de Geociências. Curso de Pós-Graduação em Geofísica - Campus Universitário do Guamá - R. Augusto Corrêa, S/N, Belém-Pará-Brasil - Fone: (+91) 2111692 - Fax: (+91) 211-1693 - E-mail: Iwbleite@ufpa.br.

2 Universidade Federal do Pará. Centro de Geociências. Curso de Pós-Graduação em Geofísica - Campus Universitário do Guamá - R. Augusto Corrêa, S/N, Belém-Pará-Brasil - Fone: (+ 91) 211 1692 - Fax: (+91) 211-1693 - E-mail: fjca@ufpa.br.
} 


\section{INTRODUÇÃOO}

0 objetivo central deste trabalho é 0 estudo dos efeitos de janelas de suavização e do tipo de fase do pulso-fonte efetivo (se mínima ou não-mínima) no desenho de filtros lineares ótimos no domínio temporal, voltado à deconvolução de dados de reflexão sísmica. Os processos envolvidos (dados e sinal desejado) são considerados estacionários, no sentido restrito, e o operador deconvolucional é tempo-invariante. Teoricamente, referimo-nos a este assunto como o problema de WienerHopf-Kolmogorov (WHK), sendo que as extensões à forma discretizada também carregam o nome de Levenberg (WHL), e a análise é ao redor das propriedades do conteúdo da informação na função autocorrelação. Os exemplos são para 0 caso monocanal, com justificativa para a geometria de afastamento-nulo, e para isto usamos o modelo de Goupillaud para simular bacias sedimentares. As limitações sobre deconvolução têm sido interessantemente abordadas por Slob e Ziolkowski (1993) e suas réplicas. Outros trabalhos em paralelo estendem 0 tratamento para operadores tempo-variante num processo nãoestacionário pelos métodos WHK e Kalman-Bucy (LEITE; ROCHA, 2000). Todas as conclusões são fortemente baseadas em simulações computacionais devido às possibilidades serem apenas numéricas (ALVES, 1999).

Sedimentologistas descrevem bacias sedimentares e os geofísicos as modelam matematicamente, ambos objetivando a exploração sísmica (KHATTRI; GIRD, 1976, entre outros). As seqüências geológicas são classificadas de muitas formas: periódica, quasi-periódica, aleatória, especial, progressiva, regressiva, etc., para substanciar uma descrição geral das suas assinaturas sísmicas. Os fenômenos físicos esperados nesses meios são descritos como devido a filmes ou a estratificação fina (BREKHOVSKY, 1980), o que corresponde a uma descrição do meio como uma função discreta (ou seja, uma função naturalmente discretizada). Neste caso, a Transformada-Z de Laplace (TZL) é uma técnica natural e conveniente para a modelagem teórica para um meio discreto, uma vez que a TLZ tem uma descrição como função discreta, enquanto que a Transformada de Laplace tem uma descrição como função contínua (ZIOLKOWSKI;FOKKEMA, 1986; BERRYMAN;GREENE;1980).

Inversão e imageamento constituem o objetivo final da maior parte dos esforços geofíisicos. A exploração sísmica pode recuperar os parâmetros elásticos, dinâmicos, e geométricos da subsuperfície como funçōes da posiçã̃o espacial. Muitas perguntas são postas quando métodos de inversão e deconvolução são aplicados a dados sísmicos de reflexão; e na literatura, deconvolução (de pulso-fonte efetivo, de múltiplas) é considerada como a primeira técnica real no processamento, com métodos e opções, através dos diferentes estágios do fluxograma do processamento, onde é desejado melhor foco nas imagens (ROBINSON; OSMAN, 1996; WAPENAAR, 1996).

A presente análise é desenvolvida com a filosofia de que a observação (isto é, dados sísmicos) é descrita como um processo aleatório com propriedades representadas pelo importante princípio de ergocidade. Por este princípio, as autocorrelações são estimadas como médias ao longo da variável temporal e, adicionalmente, é admitida a condição de estacionariedade. A condição de ergocidade é conveniente por permitir que uma mesma solução envolvendo correlações seja atacada através de um método determinístico (integral da correlação), ou por um método não-determinístico (por exemplo, correlação estocática). Sendo assim, com isto justicamos 0 intercâmbio entre estes formalismos: 0 estocástico e 0 determinístico (MARKHOUL, 1978).

Descrevemos as equações nas formas canônicas, sob a condição de discretização uniforme estabelecida na solução Goupillaud (tempoduplo unitário) que usa a TZL unilateral. As séries são decompostas em fatores binários. Por definição, fase mínima verdadeira requer que as ráizes $Z_{\mathrm{n}}$ sejam externas ao círculo unitário, $\left|z_{\mathrm{n}}\right|>1$.

\section{PROPRIEDADES DA REFLETIVIDADE}

Iniciamos a descrição com a resposta do meio ao impulso, e depois prosseguimos com a descrição do pulso-fonte efetivo para, então, montarmos o traço sísmico com base no modelo convolucional que todas tem por fundamento o teorema de Betti.

A física da propagação é regida pela equação do movimento na forma acústica 1-D:

$$
\rho(x) \partial_{t}^{2} g(x, t)=\partial_{x}\left[E(x) \partial_{x} g(x, t)\right]
$$

e o fenômeno é gerado por uma onda plana vertical e impulsiva incidente num meio formado por camadas planas horizontais, homogêneas $e$ isotrópicas. $\rho(\mathrm{x})$ é a densidade. $\mathrm{E}(\mathrm{x})$ é uma constante lástica que para ondas compressionais $\mathrm{E}(\mathrm{x})=\lambda(\mathrm{x})+2 \mu(\mathrm{x})$, e para ondas transversais $\mathrm{E}(\mathrm{x})=2 \mu(\mathrm{x})$, sendo $\lambda(\mathrm{x})$ e $\mu(\mathrm{x})$ as constantes de Lamé. $\mathrm{g}(\mathrm{x}, \mathrm{t})$ representa a amplitude da resposta transiente, $\mathrm{t}$ é a variável independente tempo: $\mathrm{x}$ é a variável independente espaço, indicando a direção de propagação. Para a elasticidade perfeita $\mathrm{E}=\rho \mathrm{c}^{2}$, sendo I= $\rho c$, a impedância acústica do meio (AKI; RICHARDS, 1980).

As condições de contorno de continuidade de deslocamento (ou pressão) e de tensão levam às definições dos coeficientes de reflexão, $\mathrm{r}_{\mathrm{k}^{\prime}}$ e de transmissão, $\mathrm{t}_{\mathrm{k}^{\prime}}$ para a interface $k$ compreendida entre as camadas $k$ e $k+1$. Para uma incidência vertical de cima para baixo, eles resultam em: 
$\mathrm{r}_{\mathrm{k}}=\left(\mathrm{I}_{\mathrm{k}}-\mathrm{I}_{\mathrm{k}+1}\right) /\left(\mathrm{I}_{\mathrm{k}}+\mathrm{I}_{\mathrm{k}+1}\right)$ e $\mathrm{t}_{\mathrm{k}}=2 \mathrm{I}_{\mathrm{k}} /\left(\mathrm{I}_{\mathrm{k}}+\mathrm{I}_{\mathrm{k}}+1\right)$.

$\mathrm{r}_{\mathrm{k}}$ e $\mathrm{t}_{\mathrm{k}}$ são números reais e $\mathrm{t}_{\mathrm{k}}+\mathrm{r}_{\mathrm{k}}=1,\left|\mathrm{r}_{\mathrm{k}}\right| \leq 1$ e $0 \leq \mathrm{t}_{\mathrm{k}} \leq 2$. Com isto, o problema físico de camadas é transformado para a física de interfaces, e os eventos contidos no traço sísmico são considerados como sendo as de reflexões primárias e secundárias (múltiplas).

A solução Goupillaud (1961) é conveniente para o caso de afastamento-nulo, e ela modela uma bacia sedimentar por um sistema de camadas uniformes (ROBINSON; TREITEL, 1980; ROBINSON, 1983). Nesta solução, 0 tempo-duplo de propagação numa camada $k$ é considerado unitário e dado por $\Delta \mathrm{t}_{\mathrm{k}}=2 \mathrm{e}_{\mathrm{k}} / \mathrm{c}_{\mathrm{k}^{\prime}}$ onde $\mathrm{e}_{\mathrm{k}}$ é a espessura e $c_{k}$ a velocidade na camada $k$. Para obter um efeito desejado com tempo de trânsito arbitrário são adicionadas interfaces sucessivas com coeficientes de reflexão zero e transmissividade unitária. As camadas são numeradas na ordem de cima para baixo, sendo $\mathrm{k}=1$ para a primeira e $\mathrm{k}=\mathrm{K}$ para a última; 0 semi-espaço superior é $0 \mathrm{k}=0$, e 0 semiespaço inferior é $\mathrm{K}+1$. As interfaces são numeradas na mesma ordem, com $\mathrm{k}=0$ para a primeira superior, e $\mathrm{k}=\mathrm{K}$ para a última inferior. Nas equações abaixo, $\mathrm{K}+1$ é reservado ao semi-espaço inferior, e a refletividade corresponde, estritamente, à resposta do sistema completo sem 'fantasma' (ghost). A discretização do semi-espaço corresponde à discretização do sinal temporal, e o método usa a TZ unilateral (condição de causalidade).

A relaçaco entre as ondas descendentes $\mathrm{D}(\mathrm{z})$, e as ascendentes $\mathrm{U}(\mathrm{z})$, e entre a parte superior $\mathrm{k}=0$ e a inferior $\mathrm{k}=\mathrm{K}+1$, é escrita na forma do propagador matricial:

$\left[\begin{array}{c}D_{K+1^{(2)}} \\ U_{K+1^{(2)}}\end{array}\right]=\frac{z^{-K / 2}}{\left(1-r_{K}\right)\left(1-r_{k-1}\right) \ldots\left(1-r_{2}\right)\left(1-r_{1}\right)}$.

$\mathrm{N}_{\mathrm{K}} \ldots \mathrm{N}_{1} \mathrm{~N}_{0}\left[\begin{array}{l}\mathrm{D}_{0}(\mathrm{z}) \\ \mathrm{U}_{0}(\mathrm{Z})\end{array}\right]$

$\mathrm{D}_{\mathrm{k}+1}(\mathrm{z})$ e $\mathrm{U}_{\mathrm{k}+1}(\mathrm{z})$ são as $\mathrm{TZ}$ dos campos descendentes $\mathrm{e}$ ascendentes $\left[d_{k+1}(t)\right.$ e $\left.u_{k+1}(t)\right]$, respectivamente, sendo a referência 0 topo da camada que neste caso indica $\mathrm{k}+1$. Nesta descrição, a fonte e os sensores são admitidos como colocados na superfície, e isto significa a base da camada $\mathrm{k}=0$. Na matriz $\mathrm{N}_{0}$ temos $\mathrm{z}=1,0$ que corresponde a $\Delta \mathrm{t}=0$ e modo a não haver retardo entre as ondas da base do semiespaço superior $(\mathrm{k}=0)$ e as do topo da camada $\mathrm{k}=1$. A amplitude da onda em $\mathrm{t}=0$ tem apenas $\mathrm{r}_{0}$ como fator.

A função transferência da reflexão para um sistema de $\mathrm{K}$ camadas é demonstrada como sendo dada por:

$\mathrm{R}(\mathrm{z})=\mathrm{R}_{\mathrm{k}}(\mathrm{z})=\frac{\mathrm{U}_{0}(\mathrm{z})}{\mathrm{D}_{0}(\mathrm{z})}=\frac{\mathrm{r}_{0} \mathrm{P}_{\mathrm{k}}(\mathrm{z})-\mathrm{Q}_{\mathrm{K}}(\mathrm{z})}{\mathrm{P}_{\mathrm{k}}(\mathrm{z})-\mathrm{r}_{0} \mathrm{Q}_{\mathrm{k}}(\mathrm{z})}$.
Os polinômios fundamentais $\mathrm{P}_{\mathrm{k}}(\mathrm{z})$ e $\mathrm{Q}_{\mathrm{k}}(\mathrm{z})$ obedecem às seguintes relações recursivas:

$P_{k}(z)=P_{k-1}(z)-r_{k} z^{k} Q_{k-1}\left(z^{-1}\right) e$

$\mathrm{Q}_{\mathrm{k}}(\mathrm{z})=\mathrm{Q}_{\mathrm{k}-1}(\mathrm{z})-\mathrm{r}_{\mathrm{k}} \mathrm{z}^{\mathrm{k}} \mathrm{P}_{\mathrm{k}-1}\left(\mathrm{z}^{-1}\right)$

As condições iniciais (em $\mathrm{k}=0$ ) são dadas por: $\mathrm{P}_{\mathrm{o}}(\mathrm{z})=\mathrm{p}_{0}(0)=1 ; \mathrm{Q}_{0}(\mathrm{z})=\mathrm{q}_{0}(0)=0.0$ denominador $\mathrm{P}_{\mathrm{k}}(\mathrm{z})$ $\mathrm{r}_{0} \mathrm{Qk}(\mathrm{z})$ tem a propriedade de ser de fase mínima, e 0 numerador $\mathrm{r}_{0} \mathrm{P}_{\mathrm{k}}(\mathrm{z})-\mathrm{Q}_{\mathrm{k}}(\mathrm{z})$ não é necessariamente de fase mínima, 0 que faz $\mathrm{R}(\mathrm{z})$ ser ou não ser de fase mínima. A divisão polinomial é ilimitada, porém, numericamente feita para corresponder ao número de camadas K. A função transferência temporal (refletividade) é oferecida pelo inverso da TZ de R(z):

$$
\begin{aligned}
& R(z)=\sum_{n=0}^{K} \varepsilon(n) z^{n}=\prod_{l=0}^{K_{i}}\left(z-z_{1}\right) \prod_{m=0}^{K_{e}}\left(z-z_{m}\right) \\
& \leftrightarrow \varepsilon(n)=\varepsilon_{M}(n) * \varepsilon_{T}(n)
\end{aligned}
$$

A expressão anterior está escrita com a decomposição de $\mathrm{R}(\mathrm{z})$ em binários, separando as ráizes em externas $\left(\mathrm{z}_{\mathrm{e}}\right)$ e internas $\left(\mathrm{z}_{\mathrm{i}}\right)$ com relação ao círculo unitário. Além disso, fizemos a correspondência com a convolução temporal entre as componentes de fase mínima $\varepsilon_{\mathrm{M}}(\mathrm{n})$ e a passa-tudo $\varepsilon_{\mathrm{T}}(\mathrm{n})$, representando a refletividade. Um dos objetivos é analisar as características de $\varepsilon(\mathrm{n})$ através da decomposição em raízes externas e internas da TZ.

Em condições especiais para análise podemos admitir que $\varepsilon(n) \approx r(n)$, ou ainda que $\varepsilon(n)=r(n)$, o que neste caso especial equivale a uma refletividade sem múltiplas (SILVIA; ROBINSON, 1979). A resposta total $\varepsilon(\mathrm{n})$ é composta do campo primário incidente e do campo secundário espalhado, e as figuras 1 e 3 mostram como 0 campo espalhado passa gradativamente a ter a mesma importância que o campo primário ao longo da resposta ao impulso.

0 estudo teórico da física de camadas finas para sísmica conta com uma vasta literatura. Além dos referenciados, incluem-se também Shapiro e Hubral (1999), Sherwood e Trorey (1965), Brridge, Papanicolaou e White (1988). Semelhantemente, algoritmos numéricos têm sido apresentados por diferentes autores: Thybo (1986), Douze (1979) e Temme e Muller (1982). 


\section{DESCRIÇÃO DO PULSO SÍSMICO}

0 modelo convolucional $1 \mathrm{D}$ para simular dados sísmicos $\mathrm{g}(\mathrm{n})$ independente do parâmetro horizontal do raio $\mathrm{p}$, é dado por: $\mathrm{g}(\mathrm{n})=\mathrm{s}(\mathrm{n})+\mathrm{v}(\mathrm{n})=\mathrm{w}(\mathrm{n}) * \varepsilon(\mathrm{n})+\mathrm{v}(\mathrm{n})$, onde $\mathrm{w}(\mathrm{n})$ representa o pulso-fonte efetivo, $\varepsilon(\mathrm{n})$ é a função refletividade, $s(n)$ é a função sinal-mensagem e $\mathrm{v}(\mathrm{n})$ é o rúido aditivo não contabilizado em $\varepsilon(n)$ ou em w(n) (Ver figuras 2A e 2B).

0 pulso-fonte efetivo $\mathrm{w}(\mathrm{n})$ pode ser descrito por várias componentes ao longo da sua trajetória na forma:

$\mathrm{w}(\mathrm{n})=\mathrm{w}_{\mathrm{O}}(\mathrm{m}, \mathrm{n}) * \mathrm{~W}_{\mathrm{F}}(\mathrm{m}, \mathrm{n}) * \mathrm{~W}^{\mathrm{A}}(\mathrm{n}) * \mathrm{~W}_{\mathrm{I}}\left(\mathrm{m}, \mathrm{n} * \mathrm{~W}_{\mathrm{E}}(\mathrm{n})\right.$.

Nesta equação estão representados os efeitos do pulso-fonte original $\mathrm{w}_{\mathrm{O}}(\mathrm{n})$ (assinatura da fonte), de múltiplas (fantasmas, não previstas na refletividade) $\mathrm{w}_{\mathrm{F}}(\mathrm{n})$, da atenuaçãa inelástica $\mathrm{w}_{\mathrm{A}}(\mathrm{n})$, do instrumento de registro $\mathrm{W}_{\mathrm{I}}(\mathrm{n})$ e da divergência esférica $\mathrm{w}_{\mathrm{E}}(\mathrm{n})$. As componentes tempo-variantes são $\mathrm{w}_{\mathrm{O}}(\mathrm{n}), \mathrm{w}_{\mathrm{F}}(\mathrm{n})$ e $\mathrm{w}_{\mathrm{T}}(\mathrm{n})$, e as componentes tempo-invariantes são $\mathrm{w}_{\mathrm{A}}(\mathrm{n})$ e $\mathrm{w}_{\mathrm{E}}(\mathrm{n})$. As componentes $\mathrm{W}_{\mathrm{F}}(\mathrm{n}), \mathrm{w}_{\mathrm{A}}(\mathrm{n})$ e $\mathrm{W}_{\mathrm{E}}(\mathrm{n})$ são consideradas fisicamente de fase mínima, $e$ as componentes $w_{O}(n)$ e $w_{I}(n)$ não são necessariamente de fase mínima. Todas essas componentes podem ser analisadas individualmente como filtros espećificos. Para os métodos de deconvolução que se baseiam nos princípios de fase mínima e de tempo-invariante, as componentes consideradas como fontes potenciais de distorção de fase são, portanto $\mathrm{w}_{\mathrm{A}}(\mathrm{n}), \mathrm{w}_{\mathrm{E}}(\mathrm{n}), \mathrm{w}_{\mathrm{O}}(\mathrm{n}), \mathrm{e} \mathrm{w}_{\mathrm{I}}(\mathrm{n})$.

Todas estas componentes do pulso podem ser submetidas a seus correspondentes operadores inversos [ $\mathrm{w}_{\mathrm{A}}^{-1}(\mathrm{n}), \mathrm{w}_{\mathrm{F}}^{-1}(\mathrm{n}), \mathrm{w}_{\mathrm{I}}^{-1}(\mathrm{n}) \mathrm{e}$ $\left.\mathrm{w}_{\mathrm{E}}^{-1}(\mathrm{n})\right]$. Na presente análise ilustrada nos restringimos a simulaçōes sem considerar separadamente os efeitos de divergência esférica, de atenuação, de instrumento e de fantasma, reunindo os efeitos na denominação de pulso-fonte efetivo, e sob condições de fase mínima e de fase não-mínima.

Devemos também incluir os efeitos de filtragens, de cirurgias e de normalizações que levam à descrição do pulso-fonte efetivo, onde estas descaracterizações são consideradas menores, porém, deformando o pulso original na direção de fase não-mínima.

\section{DESCRIÇÃO DO RUÍDO}

0 ruído pode ser descrito por uma componente ambiental [com uma parte aleatória, $\mathrm{v}_{\mathrm{aa}}(\mathrm{n})$, e uma parte coerente, $\mathrm{v}_{\mathrm{ac}}(\mathrm{n})$ ], e por uma componente relacionada à fonte sísmica [com uma parte aleatória, $\mathrm{v}_{\mathrm{fa}}(\mathrm{n})$, e uma parte coerente, $\mathrm{v}_{\mathrm{fc}}(\mathrm{n})$ ] que são submetidas à convoluçãa com pulsos filtrantes na forma: $\mathrm{v}(\mathrm{n})=\mathrm{p}_{\mathrm{va}}(\mathrm{n}) *\left[\mathrm{v}_{\mathrm{aa}}(\mathrm{n})_{-} \mathrm{v}_{\mathrm{ac}}(\mathrm{n})\right]+\mathrm{p}_{\mathrm{vf}}(\mathrm{n}) *$

$*\left[\mathrm{v}_{\mathrm{fa}}(\mathrm{n})+\mathrm{f}_{\mathrm{fc}}(\mathrm{n})\right]$.

A componente filtrante mais efetiva em $p_{v a}(n) e p_{v f}(n)$ é 0 instrumento (sensor). Mostramos aqui que a presença de ruído aditivo nos dados degrada a performance dos operadores WHL.

A deconvolução ao impulso tem como característica amplificar 0 ruído fora da banda de frequiência do pulso-fonte; como consequiência, a banda da deconvolução deve ser limitada por um filtro banda-passante equalizador que pode ser incluído como $\mathrm{w}_{\mathrm{B}}(\mathrm{n})$, definindo, com isto, 0 pulso desejado $\mathrm{w}_{\mathrm{D}}(\mathrm{n})$ na forma: $\mathrm{w}_{\mathrm{D}}(\mathrm{n})=\mathrm{w}(\mathrm{n}) * \mathrm{w}_{\mathrm{B}}(\mathrm{n})$. Além disso, 0 operador WHL obtido, h(n), bem como o seu inverso, podem ser analisados com o comprimento total (forma longa), $\mathrm{h}_{\mathrm{L}}(\mathrm{n})=\mathrm{h}(\mathrm{n})$, sendo $\mathrm{w}_{\mathrm{L}}(\mathrm{n})=\mathrm{h}_{\mathrm{L}}^{-1}(\mathrm{n})$, ou numa forma curta truncada por janela especifica $W(m), h_{C}(n)=h(n) W(n)$, sendo $w_{C}(h)=h_{C}^{-1}(n)$. Entre os objetivos definidos está de escolher um equalizador para aplicação sistemática no processo de deconvoluçãa, além da forma de truncamento para o comprimento do operador WHL (Figura 3).

\section{LIMITAÇÕES DO MODELO CONVOLUCIONAL}

0 problema fundamental na aplicação de técnicas de deconvolução em dados reais é a incerteza no conhecimento do pulsofonte efetivo, w(n). Existem muitas métodos para estimar w(n) com 0 objetivo de resolver esta ambigüidade que não é removivivel. Entre os esforços empreendidos estão os que utilizam a autocorrelação e baseiamse em modelos como 0 aqui adotado. A função autocorrelaçãao é submetida a uma janela temporal de truncamento e são várias as considerações implíitas: a) que o pulso-fonte efetivo seja estacionário e de fase mínima; b) que 0 espectro da função refletividade seja branco; e c) que 0 espectro dos dados não seja limitado a uma banda, ou que tenha sofrido distorç̃óes; d) que 0 espectro da autocorrelação destrói a fase do sinal original.

No teorema fundamental da teoria da deconvolução ótima é demonstrado que 0 operador WHL, h(n), é de fase mínima (ROBINSON; WOLD, 1962), e nesse sentido equivale a uma operação de cálculo através da transformada de Hilbert. A deconvolução preditiva - de pulso-fonte efetivo, de assinatura da fonte e de vibroseis - (ROBINSON, 1999), é baseada num pulso-fonte efetivo, $\mathrm{w}(\mathrm{n})=\mathrm{w}_{\mathrm{M}}(\mathrm{n})$ de fase mínima. Um pulso w(n), de fase não-mínima, pode ser decomposto em outro de fase mínima, $\mathrm{w}_{\mathrm{M}}(\mathrm{n})$, e um passa-tudo (deformaçãa de fase), $\mathrm{W}_{\mathrm{T}}(\mathrm{n})$, na forma $\mathrm{w}(\mathrm{n})=\mathrm{w}_{\mathrm{M}}(\mathrm{n}) * \mathrm{w}_{\mathrm{T}}(\mathrm{n})$. Por este princípio, 0 operador WH corresponde a $\mathrm{h}(\mathrm{n})=\mathrm{w}_{\mathrm{M}}^{-1}(\mathrm{n})$ e produz uma distorção de fase, $\Delta \phi(\mathrm{n})=\phi_{\mathrm{w}}(\mathrm{n})-\phi_{\mathrm{w}_{\mathrm{M}}}(\mathrm{n})$, nos dados, onde $\phi_{\mathrm{w}}(\mathrm{n})$ é 0 conteúdo de fase de $\mathrm{w}(\mathrm{n})$, e $\phi_{\mathrm{w}_{\mathrm{M}}}(\mathrm{n})$ é o conteúdo de fase de $\mathrm{w}_{\mathrm{M}}(\mathrm{n})$. 
Conhecido $w(n)$, esta propriedade resulta na determinação da componente $\mathrm{w}_{\mathrm{T}}(\mathrm{n})$ na forma: $\mathrm{h}(\mathrm{n}) * \mathrm{w}(\mathrm{n})=\mathrm{w}_{\mathrm{T}}(\mathrm{n})$.

Um pulso de fase mínima tem também um inverso de fase mínima. 0 passa-tudo tem a propriedade $\mathrm{w}_{\mathrm{T}}^{-1}(\mathrm{n})=\mathrm{w}_{\mathrm{T}}(-\mathrm{n})$, sendo, portanto, anticausal, e a convolução $\mathrm{w}_{\mathrm{T}}(\mathrm{n})$ com equivale a uma correlação e produz a compensação de fase desejada $-\Delta \phi(\mathrm{n})$. Para $\mathrm{w}(\mathrm{n})$ conhecido, o operador inverso $\mathrm{w}_{\mathrm{M}}^{-1}(\mathrm{n}) * \mathrm{w}_{\mathrm{T}}^{-1}(\mathrm{n})$ é determinado. Para 0 caso de w(n) não conhecido, é necessário uma forma independente de como estimar $\mathrm{w}(\mathrm{n})$, o que é analisado adiante (OSMAN; ROBINSON, 1996). 0 pulso-fonte é considerado como de duração relativamente curta com relação ao traço sísmico, e a sua TZ tem comprimento finito. Um dos objetivos aqui é analisar as características de $\mathrm{h}(\mathrm{n})$ através da sua decomposição em raízes da $\mathrm{TZ}$.

Podemos analisar a dificuldade existente quando a condição em que a função transferência e o pulso-fonte sejam de fase não-mínima, 0 que é expresso por:

$$
\varepsilon(\mathrm{n}) * \mathrm{~W}(\mathrm{n})=\varepsilon_{\mathrm{M}}(\mathrm{n}) * \varepsilon_{\mathrm{T}}(\mathrm{n}) * \mathrm{w}_{\mathrm{M}}(\mathrm{n}) * \mathrm{~W}_{\mathrm{T}}(\mathrm{n}) .
$$

Reagrupando em partes similares:

$$
\left[\varepsilon_{\mathrm{T}}(\mathrm{n}) * \mathrm{w}_{\mathrm{T}}(\mathrm{n})\right] *\left[\varepsilon_{\mathrm{M}}(\mathrm{n}) * \mathrm{w}_{\mathrm{M}}(\mathrm{n})\right]=\varepsilon_{\mathrm{TT}}(\mathrm{n}) * \mathrm{w}_{\mathrm{MM}}(\mathrm{n})
$$

Como conseqüência, o operador WH que resolve a parte de fase mínima destrói também a componente de fase mínima da refletividade, e a componente de fase qualquer do pulso-fonte efetivo fica inclusa na refletividade. Um dos objetivos aqui é analisar os efeitos de janelas na autocorrelação para justificar uma estimativa para $h(n)$, a partir de $\mathrm{w}(\mathrm{n})$ conhecido para casos especiais, como visto na próxima seção. Teoricamente, a operação de deconvolução sobre o traço sísmico deve ser realizada em seqüência, primeiro com a correção de fase por $\mathrm{w}_{T}^{-1}(\mathrm{n})$, e depois com $\mathrm{w}_{M}^{-1}(\mathrm{n})$. Desse modo:

$$
\mathrm{w}_{M}^{-1}(\mathrm{n}) *\left[\mathrm{w}_{\mathrm{T}}^{-1}(\mathrm{n}) * \mathrm{~g}(\mathrm{n})\right]=\varepsilon_{\mathrm{TT}}(\mathrm{n}) \approx \varepsilon(\mathrm{n}) .
$$

0 problema de deconvolução de fase misturada tem sido discutido por diversos autores: Porsani e Ursin (1998) analisam a condição especial em que as raízes da $\mathrm{TZ}$ dos pulsos não estejam no círculo unitário (conseqüentemente, os espectros de amplitude não podem ter valores nulos em qualquer freqüência). Ursin e Porsani (1999) continuam a investigação de deconvolução de fase misturada, utilizando o método de representação de um processo pelo modelo auto-regressivo, através das equações Yule-Walker. Todos os métodos que buscam remover o efeito de pulsos fora do convencional (fase mínima) requerem mais engenharia no processamento, o que pode significar incluir mais condições a priori.
Estas são situações lógicas, uma vez que as dificuldades do problema geral de deconvolução podem ser reduzidas a uma equação integral do primeiro tipo.

\section{INTERPRETAÇÃO DA FUNÇÃO REFLETIVIDADE}

Seções de reflexão sísmica de afastamento-nulo contabilizam deformações espectrais devido ao processamento de correção de sobretempo normal NMO, dependente do modelo de macro-velocidades (estiramento do pulso) e devido ao empilhamento (média sobre os traços). Sendo assim, uma componente canônica, tempo-variante, dependente do parâmetro horizontal do raio $\mathrm{p}$, digamos $\mathrm{w}_{\mathrm{NMO}}(\mathrm{p}, \mathrm{n})$, pode ser propriamente adicionada à descrição do pulso-fonte efetivo para representar adicionalmente este efeito ao longo do traço. Esta etapa do processamento é importante, e a refletividade na seção afastamentonulo deve ser considerada como uma média dentro de um intervalo de ângulos de incidência.

Além destes efeitos, falta considerar os fenômenos físicos de dispersão na transmissão-reflexão sobre a amplitude do pulso sísmico, com relação ao modelo de camadas finas.

0 resultado da deconvolução ao impulso é representado por:

$h(n) * g(n)=h(n) * w(n) * \varepsilon(n)+h(n) * v(n)$,

que pode ser reescrita por resíduos na forma:

$y(n)=w_{R}(n) * \varepsilon(n)+v_{R}(n)$.

0 operador h(n) é a estimativa do inverso do pulso-fonte efetivo $\left[\mathrm{w}(\mathrm{n})\right.$, ou $\left.\mathrm{w}_{\mathrm{D}}(\mathrm{n})\right]$ com base no algoritmo WHL, isto é, $\mathrm{h}(\mathrm{n}) \approx \mathrm{w}^{-1}(\mathrm{n}), 0$ que resulta no resíduo $\mathrm{w}_{\mathrm{R}}(\mathrm{n})=\mathrm{h}(\mathrm{n}) * \mathrm{w}(\mathrm{n})$ devido à diferença entre os sismogramas reais e teóricos abstratos.

A qualidade da imagem de saída do filtro é julgada por um interprete com base na resolução da imagem e se ela é plausível. Comparativamente, um procedimento mais sofisticado, e que demanda mais esforços, é o que busca a extração de parâmetros físicos das rochas numa distribuição espacial 3D.

\section{Forma Paramétrica da Solução WHL}

Continuamos com as equações nas formas canônicas convenientes, com a discretização uniforme estabelecida na solução Goupillaud. 0 critério para o filtro é a minimização da variância dos desvios, $\Delta \mathrm{y}(\mathrm{n})=\mathrm{d}(\mathrm{n})-\mathrm{y}(\mathrm{n})$ entre as funções $\mathrm{d}(\mathrm{n})$ (sinal desejado) e y(n) (saída real) no domínio temporal. A função objeto é dada por: 
$\mathrm{e}\left(\mathrm{h}_{\mathrm{i}}\right)=\mathrm{E}\left\{[\Delta \mathrm{y}(\mathrm{n})]^{2}\right\}$. A saída real do filtro é: $\mathrm{y}(\mathrm{n})=\mathrm{h}(\mathrm{n}) * \mathrm{~g}(\mathrm{n})$; e a minimização de $\mathrm{e}\left(\mathrm{h}_{\mathrm{i}}\right)$ resulta da equaç̧ão geral WHL:

$\sum_{\mathrm{n}} \mathrm{h}(\mathrm{n}) \phi_{\mathrm{gg}}(\mathrm{m}-\mathrm{n})=\phi_{\mathrm{dg}}(\mathrm{m})$,

e a sua solução determina os coeficientes $h(n)$. $\phi_{g g}($.$) representa a$ autocorrelação teórica da entrada, e $\phi_{\mathrm{dg}}($.$) é a correlaçã̃o cruzada$ estocástica unilateral teórica entre 0 sinal desejado e 0 sinal observado. A qualidade do filtro é aqui também medida pela fórmula do erro mínimo normalizado dado por:

$\mathrm{e}_{\min }=1-\left[\phi_{\mathrm{gg}}(0)\right]^{-1} \sum_{\mathrm{n}} \phi_{\mathrm{dg}}(\mathrm{n}) \mathrm{h}(\mathrm{n})$.

Vários problemas básicos podem ser estruturados e resolvidos a partir da equacção geral WHL. Interessa-nos aqui os sob as suas formas chamadas paramétricas (LEITE, 1998).

0 operador em foco é o da deconvolução ao impulso, cujas relações e condições a priori são definidas por: a) A entrada, $\mathrm{g}(\mathrm{n})=\mathrm{s}(\mathrm{n})+\mathrm{v}(\mathrm{n})$, onde $\mathrm{s}(\mathrm{n})$ é a mensagem e $\mathrm{v}(\mathrm{n})$ é 0 ruído aditivo; b) 0 desejado é $\mathrm{d}(\mathrm{n})=\mathrm{r}(\mathrm{n})$, e $\varepsilon(\mathrm{n})$ é considerado como uma série branca; c) A condição de nulidade da correlações cruzadas $\phi_{\mathrm{sv}}(\mathrm{n})$ e $\phi_{\mathrm{vs}}(\mathrm{n})$ resulta em

$$
\begin{gathered}
\phi_{\mathrm{gg}}(\mathrm{n})=\sigma_{\varepsilon}^{2} \phi_{\mathrm{ww}}(\mathrm{n})+\phi_{\mathrm{vv}}(\mathrm{n}) . \\
\text { Também com } \phi_{\mathrm{dg}}(\mathrm{m})=\sigma_{\varepsilon}^{2} \phi_{\mathrm{w}_{-\mathrm{m}}} \\
\text { com } \mathrm{w}_{-\mathrm{m}}=\mathrm{w}_{0} \text { para } \mathrm{m}=0, \\
\mathrm{w}_{-\mathrm{m}}=0 \text { para } \mathrm{m}>0, \\
\phi_{\mathrm{vv}}(\mathrm{n})=\sigma_{\mathrm{v}}^{2} \delta(\mathrm{n}) .
\end{gathered}
$$

A equação WHL na forma paramétrica desejada resulta em:

$$
\sum_{\mathrm{n}} \mathrm{h}(\mathrm{n})\left[\sigma_{\varepsilon}^{2} \phi_{\mathrm{ww}}(\mathrm{n})+\sigma_{\mathrm{v}}^{2} \delta(\mathrm{n})\right]=\sigma_{\varepsilon}^{2} \mathrm{w}_{-\mathrm{m}}
$$

sendo esta forma útil para análise das partes que podem contribuir na construçã̃o de um operador deconvolucional.

Um problema para comparação é o de predição cujas condições a prioris ão definidas por:

a) A entrada, $g(n)=s(n)+v(n)$, onde $s(n)$ é a mensagem e v(n) o ruído aditivo;

b) 0 desejado é $\mathrm{z}(\mathrm{n})=\mathrm{s}(\mathrm{n}+\mathrm{T})$, onde $\mathrm{T}$ é a distância de predição;

c) As correlações são resumidas a:

$\phi_{\mathrm{gg}}(\mathrm{n})=\phi_{\mathrm{ss}}(\mathrm{n})+\phi_{\mathrm{vv}}(\mathrm{n}), \phi_{\mathrm{zg}}(\mathrm{n})=\phi_{\mathrm{ss}}(\mathrm{n}-\mathrm{T}) \mathrm{e}$ $\phi_{\mathrm{vv}}(\mathrm{n})=\sigma_{\mathrm{v}}^{2} \delta(\mathrm{n})$.

A equação WH na forma paramétrica é dada por:

$\sum_{\mathrm{n}} \mathrm{h}(\mathrm{n})\left[\phi_{\mathrm{ss}}(\mathrm{n})+\sigma_{\mathrm{v}}^{2} \delta(\mathrm{n})\right]=\phi_{\mathrm{ss}}(\mathrm{m}+\mathrm{T})$.

Nesta equação, para $\mathrm{T}=0$ o problema corresponde ao de suavização, e para $\mathrm{T}=1$ o problema corresponde ao de deconvoluçãa ao impulso, a não ser por um fator constante. Na equação do problema de predição participa a autocorrelaçãa da mensagem, e no problemde deconvolução preditiva participa a autocorrelação do pulso-fonte efetivo.

As equações WHL podem ser escritas na forma compacta matricial $\underline{\underline{\Phi}} \underline{\mathrm{h}}=\underline{\Psi}$, onde $\underline{\underline{\Phi}}$ é a matriz Toeplitz formada por elementos da autocorrelaçãa $\phi_{\mathrm{gg}}(),. \Psi$ é o vetor da correlação cruzada unilateral e $\underline{\mathrm{h}}$ é 0 vetor do operador procurado. A soluçãa usada tem a forma $\underline{\mathrm{h}}=\underline{\underline{\Phi}}^{-1} \underline{\Psi}$, e utilizamos a decomposição da matriz do problema, $\underline{\underline{\Phi}}$, em valores singulares, $\underline{\underline{\Phi}}=\underline{\underline{\mathrm{U}}} \underline{\underline{\Lambda}} \underline{\mathrm{V}}^{\mathrm{T}}$, com 0 objetivo de acompanhar a condição da matriz do problema através da relação $\lambda_{\text {max }} / \lambda_{\text {min }}$ nas diferentes simulações.

A quantidade $\sigma_{v}^{2} \delta(n)$ aparece em todas as formulações básicas paramétricas e participa na diagonal da matriz $\Phi$. Como a raz̃oo sinal/ruído não é conhecida de antemão nos problemas práticos, 0 controle é através do parâmetro $\sigma_{\mathrm{v}}^{2}$, sendo a sua modificação referida como descoloração. Um dos objetivos aqui é analisar 0 efeito deste parâmetro separadamente, e de forma associada a outras partes contribuintes.

\section{JUSTIFICATIVAS DE JANELAS NA AUTOCORRELAÇÃO}

0 conhecimento de w(n) é fundamental para o processo de deconvolução. Quando isto não acontece, a deconvolução para estimar ao mesmo tempo w(n) e $\varepsilon(\mathrm{n})$ tem sido denominada deconvolução dupla (ou cega), sendo este o caso em questão. A função autocorrelação do traço sísmico, $\phi_{\mathrm{gg}}(\mathrm{n})$, pode ser expressa em termos de suas componentes e da relaçẫo entre elas. Uma dessas é a função refletividade que pode ser denominada como simples [caracterizada pela distribuição de $\mathrm{r}(\mathrm{n})$ com a condição especial de ser puramente aleatória, 0 que significa ser a autocorrelação $\phi_{\text {rr }}(n)$ uma série branca, e o que teoricamente resulta na ausência de múltiplas (SILVIA; ROBINSON, 1979)], mesmo assim $\phi_{\mathrm{gg}}(\mathrm{n})$ tem formas diversificadas. Quando a função refletividade não é do tipo simples, a autocorrelação adquire formas mais complicadas que dificultam o cálculo de operadores mais eficientes para a compressão dos eventos sísmicos, e a estas dificuldades podemos adicionar 0 efeito de rúdos. 0 comprometimento da forma da 
autocorrelação do traço sísmico pode ser analisado a partir da construção de $\mathrm{g}(\mathrm{n})$ dada anteriormente, justificado como resultante da convolução entre uma função pulso $f(n)$ e uma função refletividade $\varepsilon(n)$, sendo expresso por: $g(n)=\varepsilon(n) * f(n)+v(n)$, onde $v(n)$ é a componente ruído aditivo. A expressão geral para $\phi_{g g}(n)=E\{g(n) g(n-\eta)\} e ́$ dada por: $\phi_{\mathrm{gg}}(\mathrm{n})=\phi_{\varepsilon \varepsilon}(\mathrm{n}) * \phi_{\mathrm{ff}}(\mathrm{n})$, onde os termos cruzados com a componente ruído são considerados não-correlacionáveis. Para a condição especial de $\phi_{\varepsilon \varepsilon}(n)$ como uma série branca, então $\phi_{\varepsilon \varepsilon}(n) \sigma_{\varepsilon}^{2} \delta(n)$, onde $\delta(n)$ é o delta de Dirac, e $\phi_{\mathrm{gg}}(\mathrm{n})$ resulta numa simples forma escalonada de $\phi_{\mathrm{ff}}(\mathrm{n})$ com $\phi_{\mathrm{gg}}(\mathrm{n}) \sigma_{\varepsilon}^{2} \phi_{\mathrm{ff}}(\mathrm{n})$. Sob esta condição, algumas características de $\phi_{\mathrm{gg}}(\mathrm{n})$ podem ser analisadas através de $\phi_{\mathrm{ff}}(\mathrm{n})$, e podemos fazer $\sigma_{\varepsilon}^{2}=1$ para simplificação. Um exemplo intencionalmente simples é o caso de uma múltipla simples, não contabilizada em $\varepsilon(n)$, que é representado por um pulso retardado de $\mathrm{T}$ unidades, sendo o pulso-fonte construído por:

$f(n)=w(n)-r w(n-T)$

$\mathrm{T}=$ (espessura da camada)/(velocidade da camada), e r é o coeficiente de reflexão entre a camada e o semi-espaço inferior. A autocorrelação resulta em:

$\phi_{\mathrm{ff}}(\mathrm{n})=\left(1+\mathrm{r}^{2}\right) \phi_{\mathrm{ww}}(\mathrm{n})-\mathrm{r}\left[\phi_{\mathrm{ww}}(\mathrm{n}+\mathrm{T})+\phi_{\mathrm{ww}}(\mathrm{n}-\mathrm{T})\right]$,

que é uma superposição da função $\phi_{\text {ww }}(n)$ com fatores de atenuaçãa de amplitudes, e com máximos em simetria localizados em $\phi(0)$, $\phi(-\mathrm{T})$ e $\phi(+\mathrm{T})$. Um próximo caso é o de múltiplas simples com deslocamento distintos $\left(\mathrm{T}^{1}\right.$ e $\left.\mathrm{T}^{2}\right)$, descrito por:

$f(n)=w(n)-r_{1} w\left(n-T_{1}\right)-r_{2} w\left(n-T_{2}\right)$.

A autocorrelação resulta em:

$\phi_{\mathrm{ff}}(\mathrm{n})=\left(1+\tau_{1}^{2}+\mathrm{r}_{2}^{2}\right) \phi_{\mathrm{ww}}(\mathrm{n})-\tau_{1} \mathrm{r}_{2}$

$\left[\phi_{\mathrm{ww}}\left(\mathrm{n}+\mathrm{T}_{1}-\mathrm{T}_{2}\right)+\phi_{\mathrm{ww}}\left(\mathrm{n}-\mathrm{T}_{1}+\mathrm{T}_{2}\right)\right]-$

$-\tau_{1}\left[\phi_{\mathrm{ww}}\left(\mathrm{n}-\mathrm{T}_{1}\right)+\phi_{\mathrm{ww}}\left(\mathrm{n}+\mathrm{T}_{1}\right)\right]-$

$\mathrm{r}_{2}\left[\phi_{\mathrm{ww}}\left(\mathrm{n}-\mathrm{T}_{2}\right)+\phi_{\mathrm{ww}}\left(\mathrm{n}+\mathrm{T}_{2}\right)\right]$

que é novamente a superposição de $\phi_{\text {ww }}(n)$ com fatores de amplitude e deslocamentos distintos, e máximos de $\phi_{\mathrm{ff}}(\mathrm{n})$ em $\mathrm{n}=\left\{0, \mathrm{~T}_{1},-\mathrm{T}_{1}, \mathrm{~T}_{2},-\mathrm{T}_{2},-\left(\mathrm{T}_{1}-\mathrm{T}_{2}\right),+\left(\mathrm{T}_{1}-\mathrm{T}_{2}\right)\right\}$.

Uma generalização aos dois casos acima é transferir múltiplas para 0 pulso-fonte efetivo através da seguinte função:
$f(n)=\sum_{i=0}^{I} r_{i} w\left(n-T_{i}\right)$.

0 resultado para a autocorrelação é que:

$\phi_{\mathrm{gg}}(\mathrm{n}) \approx \phi_{\mathrm{ff}}(\mathrm{n}) \sum_{\mathrm{i}=0}^{\mathrm{I}} \sum_{\mathrm{l}=0}^{\mathrm{I}} \mathrm{r}_{\mathrm{i}} \mathrm{r}_{1} \phi_{\mathrm{ww}}\left(\mathrm{n}-\mathrm{T}_{\mathrm{i}}+\mathrm{T}_{1}\right)$.

0 primeiro termo da serie é $\sigma_{\mathrm{r}}^{2} \phi_{\mathrm{ww}}(\mathrm{n})$, onde a variância $\sigma_{\mathrm{r}}^{2}=\sum_{\mathrm{i}=0}^{\mathrm{I}} \mathrm{r}_{\mathrm{i}}^{2}$ modifica naturalmente 0 segmento desejado $\phi_{\mathrm{ww}}(\mathrm{n})$, e 0 operador deconvolucional tem embutido um fator que escalona 0 desejado $\varepsilon(n)$. Disto, concluímos que para selecionar $\phi_{w w}(n)$ é necessário que o comprimento do pulso-fonte e da janela temporal de truncamento e suavização não contenham a primeira múltipla considerada distanciada de $\mathrm{T}_{1}$. Conseqüentemente, 0 operador com comprimento alongado incluindo $\mathrm{T}_{1}$ deconvolve a múltipla $\mathrm{T}_{1}$, e 0 operador de comprimento incluindo $\mathrm{T}_{1}+\mathrm{T}_{2}$ deconvolve as múltiplas $\mathrm{T}_{1}$ e $\mathrm{T}_{2^{\prime}}$ e assim por diante. 0 operador não discerne a múltipla do real. Estes modelos simples são exemplos da estrutura da autocorrelaçãa. As distâncias entre máximos de $\phi_{\mathrm{gg}}(\mathrm{n})$ permitem, no princípio, a medição de parâmetros importantes ( $\mathrm{T}, \mathrm{T}_{1}$ e $\mathrm{T}_{2}$ ) para 0 modelo. A solução Goupillaud também permite uma interpretação física para a autocorrelação, o que reforça o seu enfoque na análise dos operadores ótimos (CLAERBOUT, 1968; ROBINSON, 1983).

Uma janela temporal conveniente para a autocorrelação é a função exponencial com decaimento $\beta$ expresso por:

$W(n)=\left\{\begin{array}{ll}\mathrm{e}^{-\beta|n|},|n|=0,1,2, \ldots, J-1 \\ 0, & ,|n| \geq J\end{array}\right.$.

A aplicação de uma janela na autocorrelaçãa $\phi_{\mathrm{gg}}(\mathrm{n})$ tem a forma $\phi_{\mathrm{gg}}^{\mathrm{w}}(\mathrm{n})=\phi_{\mathrm{gg}}(\mathrm{n}) \mathrm{W}(\mathrm{n})$. Como conseqüência dessa argumentação e as das seções anteriores, o princípio é obter forçosamente 0 operador $h(n)$ a partir da autocorrelação $\phi_{\mathrm{gg}}^{\mathrm{w}}(\mathrm{n})$, cuja resolução é medida visualmente pela melhor forma impulsiva representada de forma canônica por $\mathrm{h}(\mathrm{n}){ }^{*} \mathrm{w}(\mathrm{n}) \approx \delta(\mathrm{n})$. 0 comprimento unilateral da autocorrelação resultante da otimização com a janela retangular pode ser 0 critério para estabelecer o comprimento de w(n) no traço original. Outra forma é acoplar técnicas diretas para recuperação de w(n), como a transformada de Hilbert e a análise homomórfica coletados em Osman e Robinson (1996).

\section{Exemplos Selecionados}

As figuras selecionadas para apresentação são parciais (com relação às produzidas para o presente trabalho). No entanto, as conclusões gerais referem-se a todo o álbum, descrito em resumo, a seguir, de 
forma seqüencial. Com 0 intuito de obter operadores mais eficientes a partir de informaçôes contidas em $\phi_{\mathrm{gg}}(\mathrm{n})$ e numa forma mais prática, montamos uma sistemática de análise com base em resultados numéricos, uma vez que soluções fechadas não parecem simples.

As argumentações entre os objetivos e as conclusões são sobre os seguintes efeitos: a) das janelas de truncamento e de suavização (figuras 4, 7, 10, 13); b) do tipo de fase do operador (fase mínima ou fase não-mínima); c) da medida de qualidade (figuras 4.f, 5, 7.f, 8 , 10.f, 11, 13.f, 14, 15, 16.f, 17.f); d) dos ruídos presentes e da equalização (figuras 10 a 17); e) da condição de traço balanceado (figuras 16 e 17); f) do nível de descoloração (figuras 10 a 17).

0 valor da raz̃̃o sinal/ruído (S/R) neste trabalho é definido pela razão entre variância estatística definida por:

$\sigma_{\mathrm{x}}^{2}=\mathrm{V}\{\mathrm{x}(\mathrm{n})\}=\frac{1}{\mathrm{~N}} \sum_{\mathrm{n}=1}^{\mathrm{N}}[\mathrm{x}(\mathrm{n})-\overline{\mathrm{x}}]$,

$\bar{x}=\frac{1}{N} \sum_{n=1}^{N} x(n)$.

$\mathrm{X}(\mathrm{n})$ representa as componentes do sinal para 0 cálculo de diferentes definições do quociente $S / R$ : a) mensagem/ruído, $S_{s} / R_{v}=\sigma_{s}^{2} / \sigma_{v}^{2}$; b) (mensagem com rúido)/ruído, $\mathrm{S}_{\mathrm{g}} / \mathrm{R}_{\mathrm{v}}=\sigma_{\mathrm{g}}^{2} / \sigma_{\mathrm{v}}^{2}$; c) (mensagem com ruído)/filtrado $\mathrm{S}_{\mathrm{g}} / \mathrm{R}_{\mathrm{y}}=\sigma_{\mathrm{g}}^{2} / \sigma_{\mathrm{y}}^{2}$; d) e em níveis de decibéis, $\mathrm{Xdb}=10 \log _{10}\left(\sigma_{1}^{2} / \sigma_{2}^{2}\right)$.

Filtros de equalização são aplicados na presença de níveis diferentes de rú́do nos dados, e optamos pelo denominado passa-baixa de Ormsby (PBO), com as freqüências de corte (baixa/alta) informadas pelos índices de numeração do espectro (exemplo, 80/120).

As figuras são agrupadas em blocos com dificuldades crescentes. No Bloco A temos as figuras 1, 2A, 2B e 3 que mostram uma distribuição dos coeficientes de reflexão, um exemplo de pulso-fonte, a resposta ao impulso, e os sismogramas resultantes utilizados nos experimentos. As autocorrelaçõoes servem para indicar o desvio da condição de série branca da respectiva distribuiçã̃o. Os espectros de amplitude e de fase restaurada são informações qualitativas do conteúdo bandeado destas funções para orientar no desenho do equalizador. As raízes da TZ informam a condição de fase mínima. Nos casos de simulações simples os resultados são bem evidentes. Porém, os exemplos selecionados são casos mais elaborados. A análise das figuras e as conclusões devem ter base na teoria.

№ Bloco B temos as figuras 4, 5 e 6 que formam os exemplos dos efeitos de janelas selecionadas sobre as autocorrelações dos sismogramas sem ruído e com um pulso-fonte efetivo de fase mínima. A qualidade é verificada através da medida da função erro, e também pela operação de convolução com o operador inverso. 0 Bloco C contém as figuras 7,8 e 9 e segue a mesma sequiencia do Bloco B, sismogramas sem ruído, mas com um pulso-fonte efetivo de fase não-mínima. 0 Bloco $D$ contém as figuras 10, 11 e 12 e segue a mesma sequênncia dos Blocos B e C, porém com sismogramas com ruído e com pulso-fonte efetivo de fase mínima, e com aplicação de equalizador. 0 Bloco $E$ contém as figuras 13,14 e 15 e segue a mesma sequiência dos blocos B, C e D, porém, com sismogramas com ruído e com pulso-fonte efetivo de fase não-mínima e aplicação de equalizador. 0 Bloco $\mathrm{F}$ contém as figuras 16 e 17 com exemplos de balanceamento dinâmico, dos efeitos do equalizador e de descoloração sobre traços selecionados das figuras dos blocos anteriores.

\section{CONCLUSÕES GERAIS}

1) Para selecionar $\phi_{w w}(\tau)$ é necessário que o comprimento do pulsofonte e da janela temporal de truncamento e suavização atenue a partir da primeira múltipla descendente distanciada de um certo valor $\mathrm{T}_{1}$. Uma múltipla ascendente (fantasma), não contabilizada no modelo, deve ficar contida como parte do pulso-efetivo.

2) 0 operador inverso contém um fator de escalonamento proporcional à variância da refletividade. Pela escala de plotagem dos resultados das convoluções não dá para observar o efeito do fator variância da refletividade na forma $\left(1+\sigma_{\mathrm{r}}^{2}\right)$ nas amplitudes recuperadas.

3) 0 tipo de fase (se mínima ou não-mínima) do operador deconvolucional deve ser verificado sistematicamente.

4) A condição da matriz Toeplitz medida pela razão dos autovalores $\left(\lambda_{\text {max }} / \lambda_{\text {min }}\right)$ mostra que o problema é bastante estável sem haver necessidade de truncamento de autovalores.

5) A presença de ruído fora da banda do pulso-efetivo faz com que seja necessário um filtro equalizador do tipo passa-baixa, ou do tipo bandapassante.

6) A aplicação de descoloraçã̃o atua com um efeito passa-baixa, e a sua aplicação deve ser acoplada ao equalizador.

7) A deconvolução é degenerada gradativamente ao passo que as raízes do pulso-fonte efetivo passam a estar posicionadas internamente ao ćírulo unitário da $\mathrm{TZ}$.

8) Não é possível separar entre as raízes (no domínio-Z) que pertencem ao pulso-fonte efetivo daquelas da refletividade. Melhor proposta para tratar o problema requer a priorimais condições baseadas em modelos da propagação de ondas elásticas e da teoria da comunicação.

\section{Agradecimentos}

Os autores agradecem ao Prof. Dr. Enders A. Robinson, pelas sugestões e comentários para o desenvolvimento deste artigo, e estendem 
os agradecimentos aos "referees" ocultos pelas sugestões úteis. 0 primeiro autor agradece a CAPES pela bolsa de pós-doutorado no exterior (Universidade de Karlsruhe, Instituto de Geofísica, Alemanha), e ao WITConsorcium, na pessoa do Prof. Dr. Peter H. W. Hubral, pelas discussões cientificas e apoio no ano sabático acadêmico, o que contribuiu para a conclusão deste trabalho. Agradecemos também 0 apoio financeiro do projeto PRH-ANP/MME/MCT-UFPa, que possibilitou os trabalhos de pesquisa.

\section{REFERÊNCIAS}

AKI, K.; RICHARDS, P. G. Quantitative Seismology. New York: Freeman, 1980. v. 1 and 2.

ALVES, F. J. C. Avaliação do efeito da janela e descoloração nos filtros Wiener-Hopf. 1999. Dissertação (Mestrado)-Curso de Pós-graduação em Geofísica, Universidade Federal do Pará, Belém, 1999. p. 1-145.

BERRYMAN, J. G.; GREENE, R. R. Discrete inverse methods for elastic waves in layered media. Geophysics, [S.I.], v. 45, n. 2, p. 213-233, 1980.

BREKHOVSKY, L. M. Waves in layered media. New York: Academic Press, 1980.

BURRIDGE, R.; PAPANICOLAOU, G. S.; WHITE, B. S. One-dimensional wave propagation in a highly discontinuous medium. Wave Motion, [S.I.], v. 10, n. 1, p. 19-44, 1988.

CLAERBOUT, J. F. Synthesis of a layered medium from its acoustic transmission response. Geophysics, [S.I.], v. 33, n. 2, p. 264-269, 1968. DOUZE, E. J. Synthetic seismograms at non-vertical incidence. Geophysical Prospecting, [S.I.], v. 27, p. 564-575, 1979.

GOUPILLAUD, P. L. An approach to inverse filtering of near-surface layer effects from seismic records. Geophysics, [S.I.], v. 26, n. 6, p. 754-760, 1961.

KHATTRI, K.; GIRD, R. A study of the seismic signatures of sedimentary models using synthetic seismograms. Geophysical Prospecting, [S.I.], v. 24, n. 3, p. 454-477, 1976.

LEITE, L. W. B.; ROCHA, M. P. C. Deconvolução de processo sísmico nãoestacionário. Revista Brasileira de Geofísica, São Paulo, v. 18, n. 1, p. 75-89, 2000.

1998.
MAKHOUL, J. Linear prediction: a tutorial review. In: CHILDERS, D. G. (Ed.). Modern spectral analysis. New York: IEEE Press, 1978. p. 1-333. OSMAN, 0. M.; ROBINSON, A. E. (Ed.). Seismic source signature estimation and measurment. Tulsa: Society of Exploration, 1996.

PORSANI, M. J.; URSIN, B. Mixed-phase deconvolution. Geophysics, [S.I.], v. 63, n. 2, p. 637-647, 1998.

ROBINSON, A. E. Multichannel time series analysis with digital computer programs. 2. ed. Houston: Goose Pond Press, 1983.

Seismic inversion and deconvolution. Part B: dual-sensor

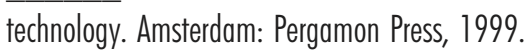

; OSMAN, 0. M. (Ed.). Deconvolution 2. Tulsa: Society of Exploration, 1996. Hall, 1980.

; TREITEL, S. Geophysical signal analysis. New Jersey: Prentice-

; WOLD, H. Structural properties of stationary stochastic processes with applications. 1962. Proceedings of the Symposium on Time Series Analysis at Brown University, New York, 1962.

SHAPIRO, S. A.; HUBRAL, P. Elastic waves in random media. Berlin: Springer-Verlag, 1999.

SHERWOOD, J. W. C.; TROREY, A. W. Minimum phase and related properties of the response of a horizontally stratified absorptive earth to plane acoustic waves. Geophysics, [S.I.], v. 30, n. 2, p. 191-197, 1965. SILVIA, M. T.; ROBINSON, E. A. Deconvolution of geophysical time series in the exploration for oil and natural gas. Amsterdam: Elsevier, 1979. SLOB, E.; ZIOLSKOWSKI, A. Aspects of 1D seismic modeling using the Goupillaud principle. Geophysical Prospecting, [S.I.], v. 41, p. 135-148, 1993.

TEMME, P.; MULLER, G. Numerical simulation of vertical seismic profiling. Journal of Geophysics, [S.I.], v. 50, p. 177-188, 1982.

THYBO, H. An algorithm for fast time-domain computation of onedimensional synthetic vertical seismic profiles. Geophysical Prospecting, [S.I.], v. 34, p. 833-844, 1986.

URSIN, B.; PORSANI, M. J. Estimation of an optimal mixed phase inverse filter. 1999. In press.

WAPENAAR, C. P. A. Inversion versus migration: a new perspective to an old discussion. Geophysics, [S.I.], v. 61, n. 3, p. 804-814, 1996.

ZIOLKOWSKI, A.; FOKKEMA, J. T. Tutorial. The progressive attenuation of high-frequency energy in seismic reflection data. Geophysical Prospecting, [S.I.], v. 34, p. 981-1001, 1986. 
a)

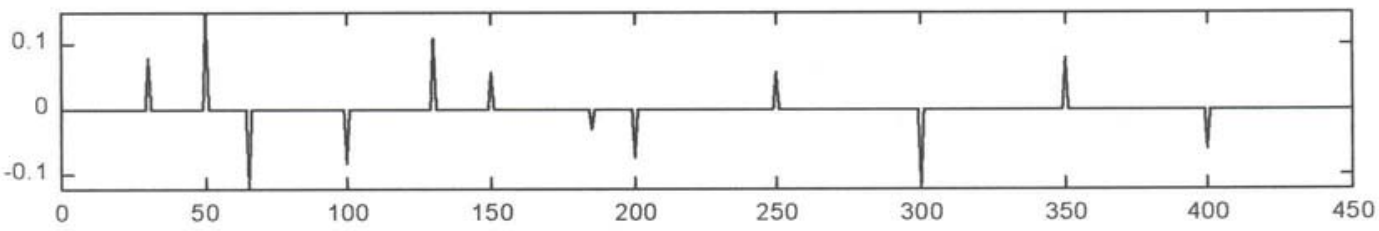

Índice, $n$

b)

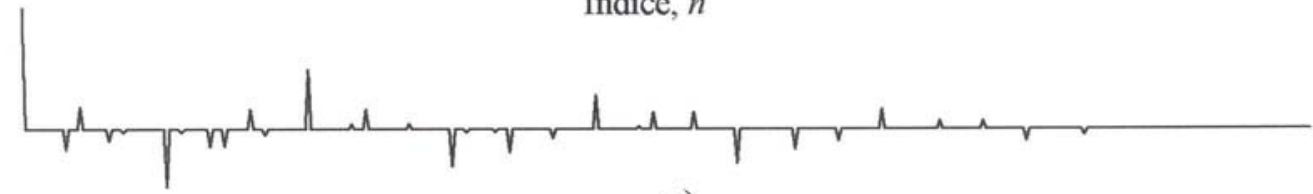

c)

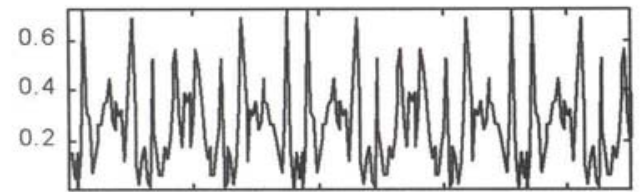

e)
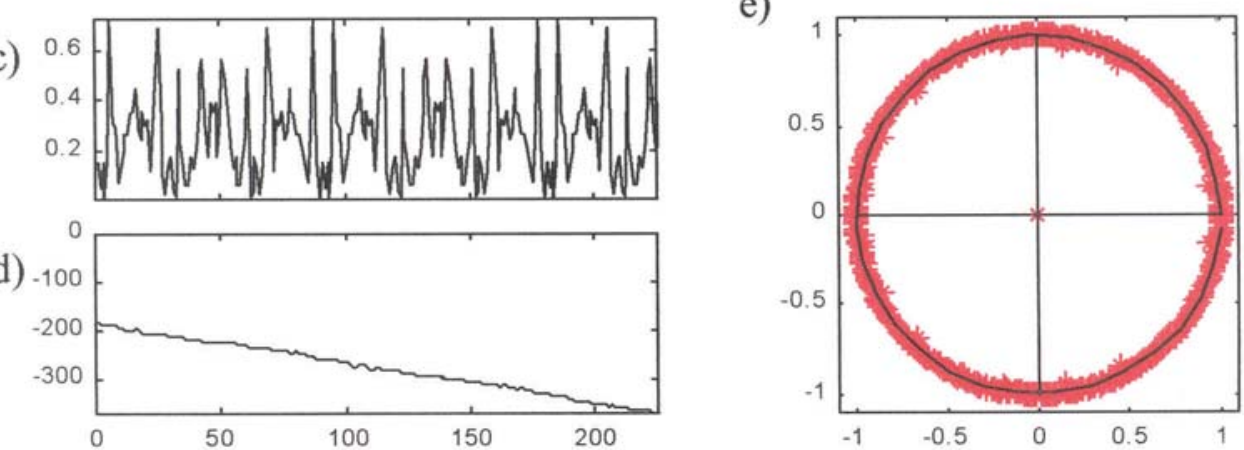

Índice, $n$

Raízes

Figura 1 - (Bloco A). (a) Distribuição dos coeficientes de reflexão para as simulações. (b) Autocorrelação mostrando desvio à série branca. (c) Espectro de amplitude. (d) Espectro de fase. (e) Ráizes da TZ da distribuição mostrando a característica de fase não-mínima (fase misturada), e as ráizes distribuídas uniformemente ao redor do círculo unitário.

Figure I - (Block A). (a) Distribution of reflection coefficients for the numerical simulations. (b) Autocorrelation showing deviation from a white series. (c) Amplitude spectrum. (d) Phase spectrum. (e) Roots of the ZT of the distribution showing the non-minimum phase(mixed-phase) characteristics, and the roots distributed uniformly around the unitary circle. 
a)

c)
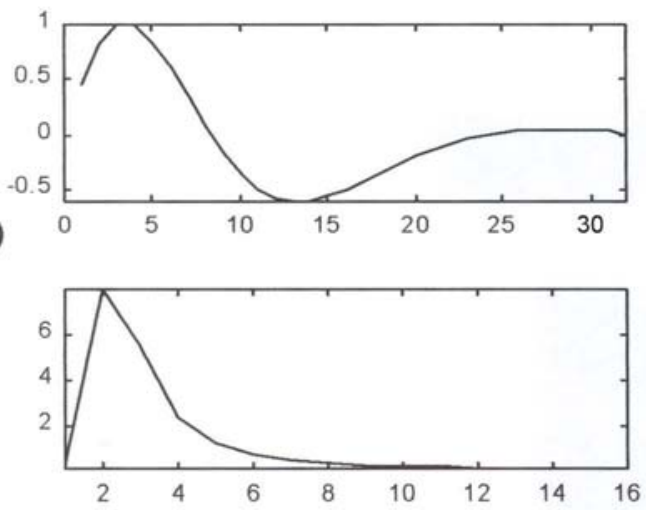

b)
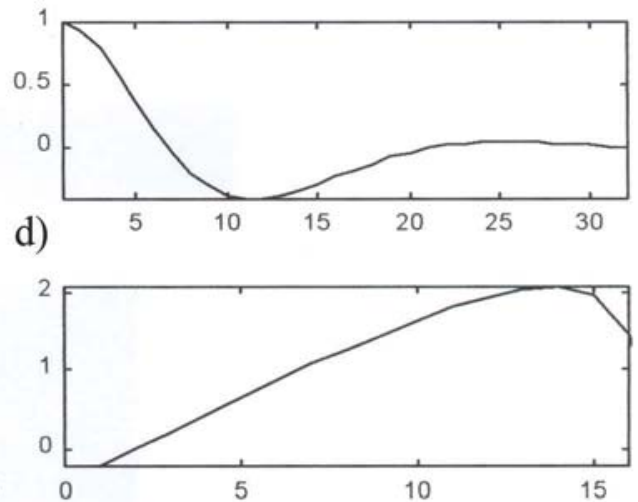

Índice, $n$

e)

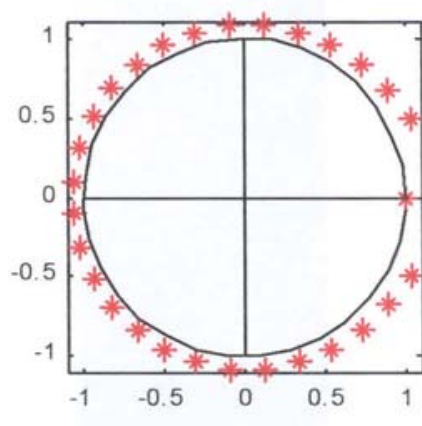

Índice, $n$

Raizes

Figura 2A - (Bloco A). (a) Exemplo do pulso-fonte original modelado pela função Berlage, fase mínima e sem rúído. (b) Autocorrelaçãao do pulso. (c) Espectro de amplitude. (d) Espectro de fase. (e) Ráizes da TZ indicando a condição de fase mínima devido as ráizes estarem no exterior do círculo unitário.

Figure 2A - (Block A). (a) Example of the original source wavelet modeled by the Berlage function, minimum phase and without noise. (b) Autocorrelation of the wavelet. (c) Amplitude spectrum. (d) Phase spectrum. (e) Roots of the IT indicating the condition of minimum phase due to the roots being placed only in the exterior of the unitary circle. 
a)

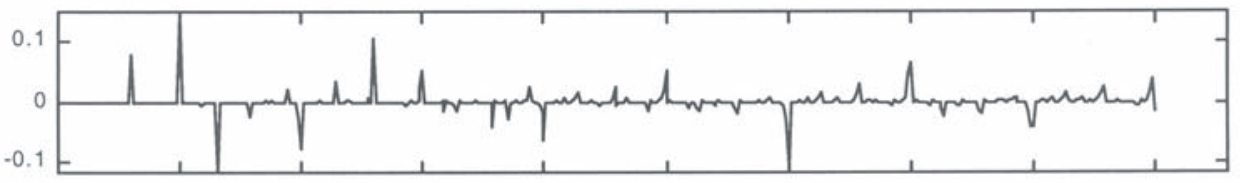

b)

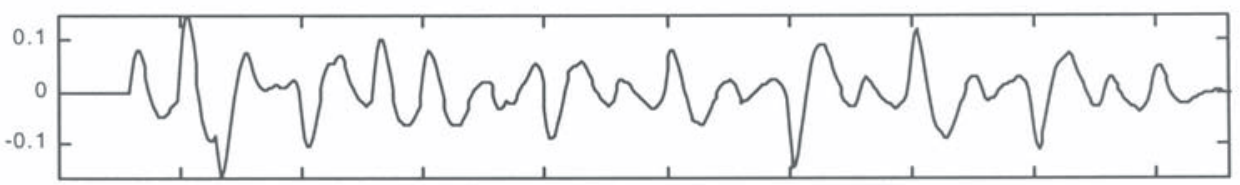

c)

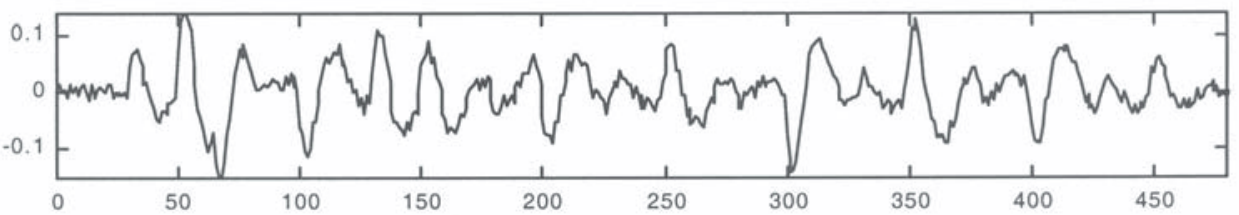

d)

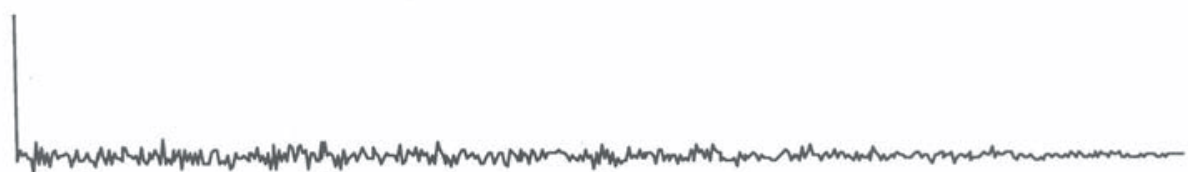

Índice, $n$

Figura 3 - (Bloco A). a) Exemplo de resposta ao impulso pela solução Goupillaud usada em todos as simulações. b) Sismograma sem ruído. c) Sismograma com ruído. d) Autocorrelaçãa do ruído.

Figure 3 - (Block A). a) Example of an impulse response by the Goupillaud solution used in all simulations. b) Seismogram without noise. c) Seismogram with noise. d) Autocorrelation of noise. 
a)

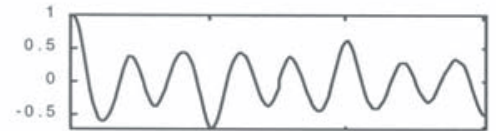

b)

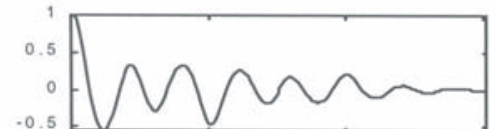

c)

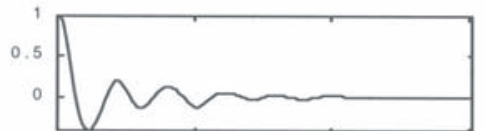

d)

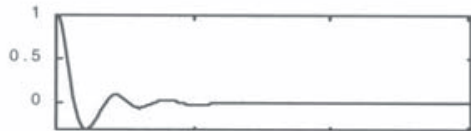

e)

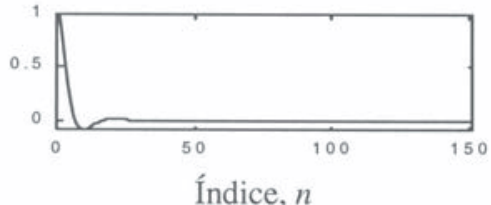

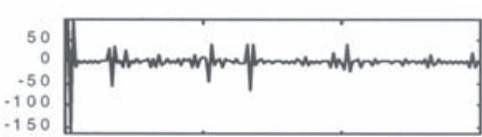
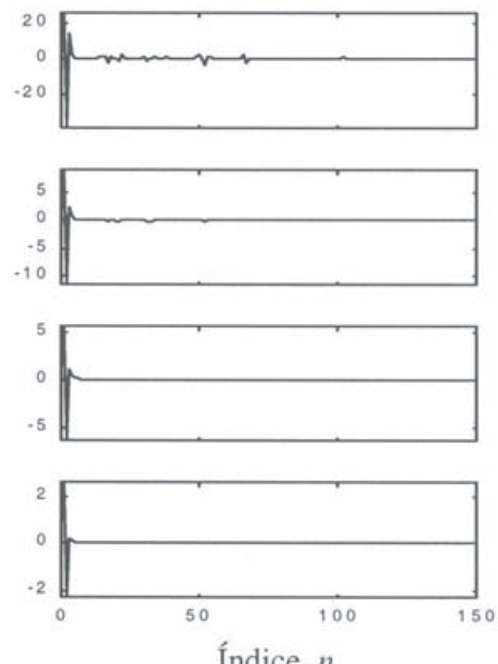

Índice, $n$
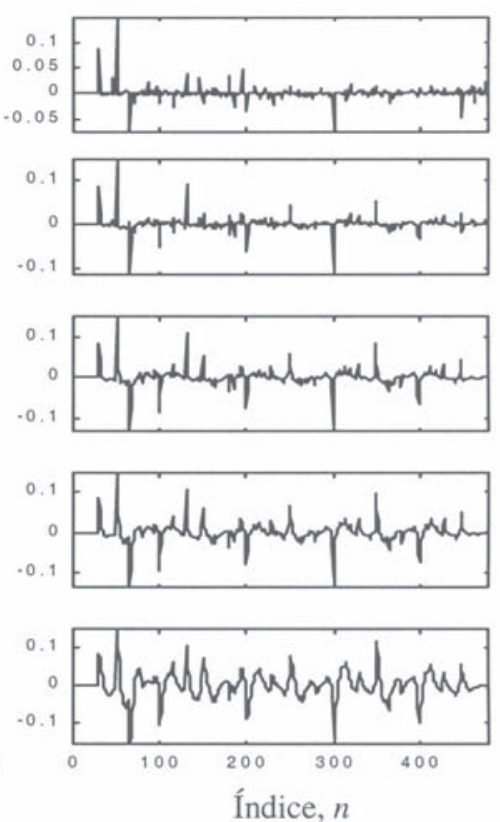

f)

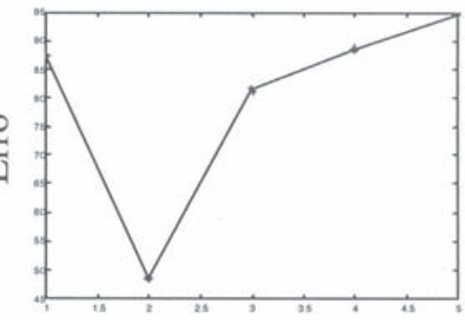

Operadores a, b, c, d,e.

Figura 4 - (Bloco B). Caso fase mínima e sem ruído. A coluna esquerda mostra autocorrelaçóes sob janelas diferentes, a coluna central os operadores longos, $\mathrm{h}_{\mathrm{b}}(\mathrm{n})=\mathrm{h}(\mathrm{n})$, obtidos, e a coluna direita os sismogramas deconvolvidos. a) Janela retangular. b) Janela triangular. c) Janela exponencial com decaimento 30 . d) Janela exponencial com decaimento 15. e) Janela exponencial com decaimento 5. f) A parte inferior mostra a variação do erro do operador para as diferentes janelas. Em paralelo, as ráizes $Z_{n}$ dos operadores foram analisadas (mas não mostradas), e elas estão sistematicamente externas ao círculo unitário, uniformemente distribuídas e progressivamente mais distanciadas. A melhor solução é ao redor de b).

Figure 4 - (Block B). Case of minimum phase without noise. The leff column shows the autocorrelations under different windows, the central column shows the long operators, $\mathrm{h}_{\mathrm{L}}(\mathrm{n})=\mathrm{h}(\mathrm{n})$, obtained, and the right column shows the deconvolved seismograms. a) Rectangular window. b) Triangular window. c) Exponential window with decay 30. d) Exponential window with decay 15. e) Exponential window with decay 5. f) The lower part shows the variation of the error of the operator for different windows. In parallel, the $\mathrm{z}_{n}$ roots of the operators were analyzed (but not shown), and they are systematically external to the unitary circle, uniform/y distributed and progressively distanciated. The best solution is around b). 


$$
h^{-1}(n) \text { e } w_{C}(n)
$$

a)

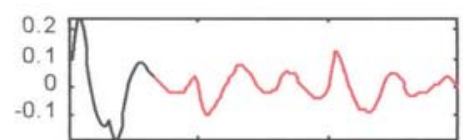

b)

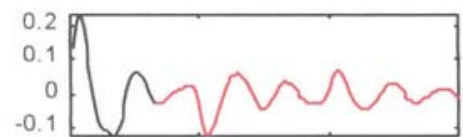

c)

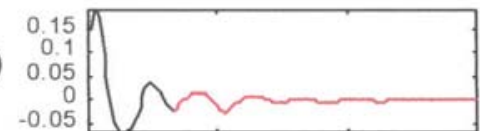

d)

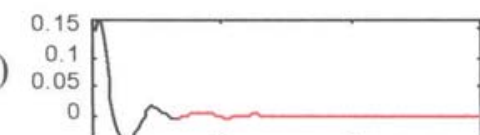

e)

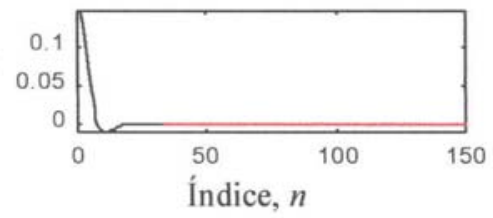

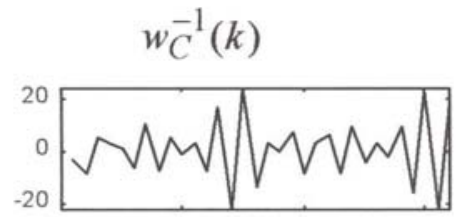
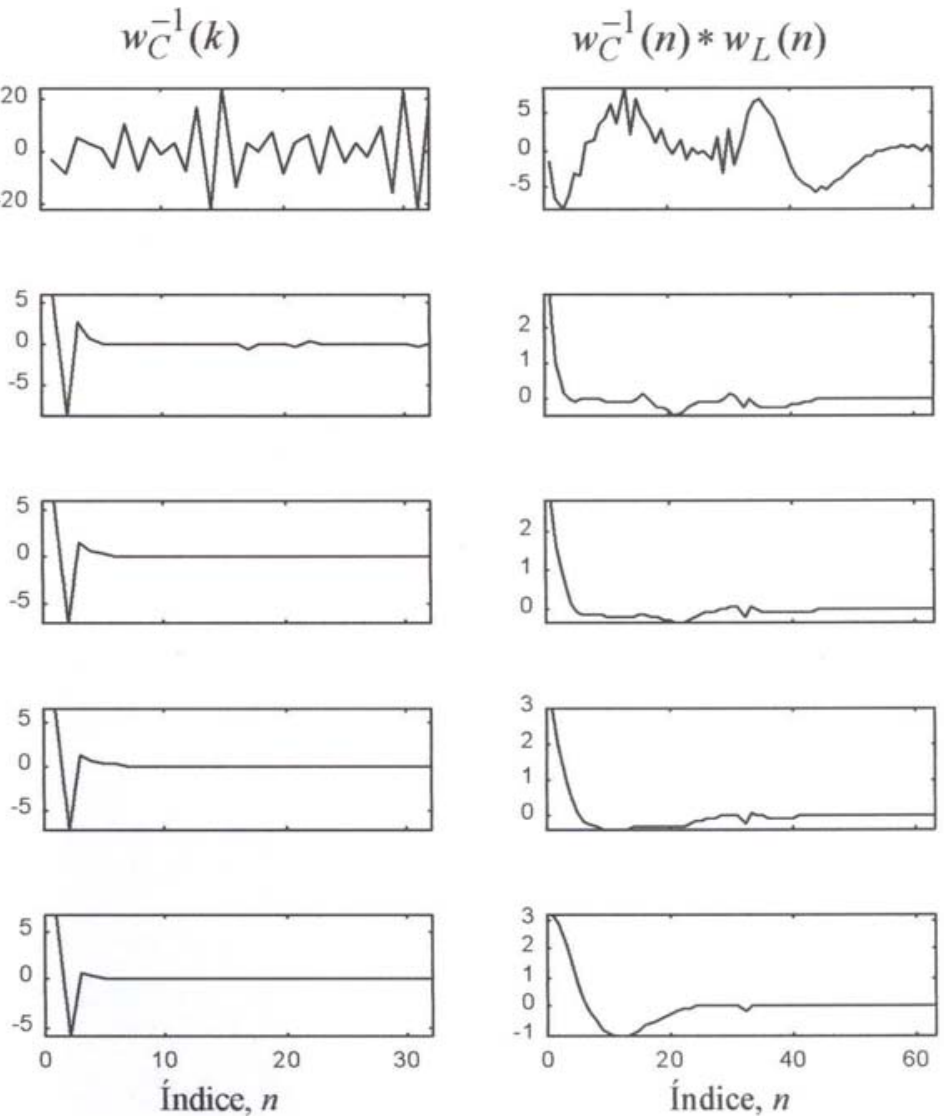

Figura 5 - (Bloco B). Caso fase mínima e sem ruído. Análise da qualidade dos operadores da Figura 4. A coluna esquerda mostra

$h^{-1}(n)=w_{L}(n)$ e $w_{C}(n), a$ coluna central $w_{C}^{-1}(n)$ e a coluna direita $w_{C}^{-1}(n) * w_{L}(n)$. As letras a), b), c), d) e e) seguem a mesma sequênncia da Figura 4. A melhor solução visual é ao redor de b) e c).

Figure 5 - (Block B). Case of minimum phase without noise. Quality analysis of the operators shown in Figure 4. The left column shows

$\mathrm{h}^{-1}(\mathrm{n})=\mathrm{w}_{\mathrm{L}}(\mathrm{n})$ and $\mathrm{w}_{\mathrm{C}}(\mathrm{n})$, the central column shows $\mathrm{w}_{\mathrm{C}}^{-1}(\mathrm{n})$, and the right column shows $\mathrm{w}_{\mathrm{C}}^{-1}(\mathrm{n}) * \mathrm{w}_{\mathrm{L}}(\mathrm{n})$. The letters a), b), c), d) and e) follow the same sequence as in Figure 4 . The best visual solution is around b) and $c$ ). 
a)

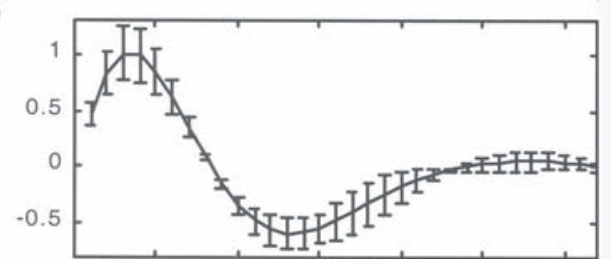

c)

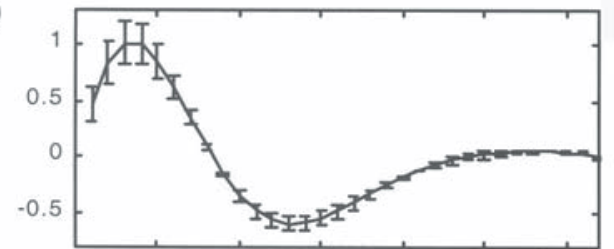

e)

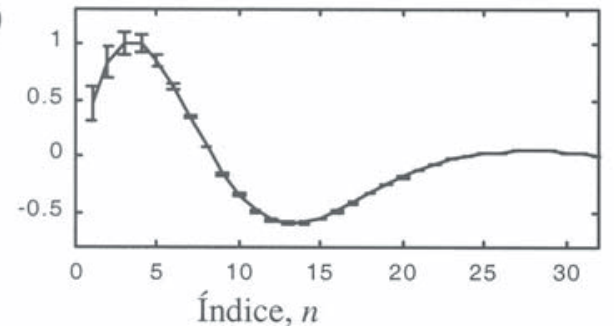

b)

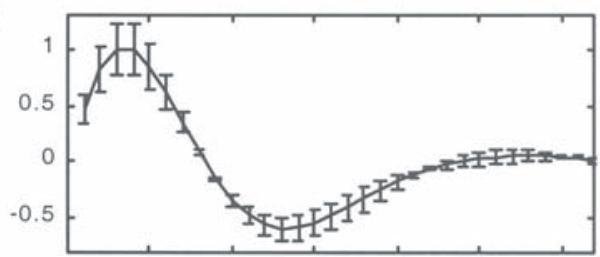

d)

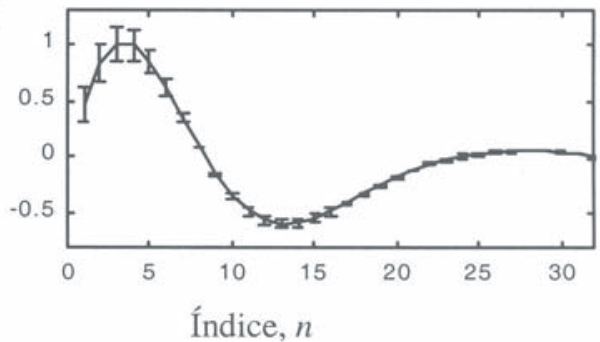

Figura 6 - (Bloco B). Caso fase mínima e sem ruído. Pulsos-fontes efetivos curtos, $\mathrm{w}_{\mathrm{C}}(\mathrm{n})$, recuperados pelos operadores exibidos nas Figuras 4 e 5. A linha continua é o pulso original, e as barras indicam a diferença para o pulso recuperado. As letras a), b), c), d) e e) seguem a mesma sequiência das Figuras 4 e 5 .

Figure 6 - (Block B). Case of minimum phase without noise. Effective short source wavelet, $\mathrm{w}_{\mathrm{C}}(\mathrm{n})$, recovered by the operators shown in Figures 4 and 5. The continuos line is the original wavelet, and the bars indicate the difference from the recovered wavelets. The (etters a), b), c), d) and e) follow the same sequence as in Figures 4 and 5. 
a)
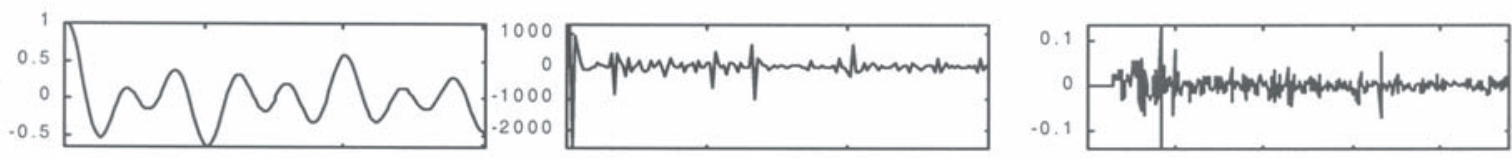

b)
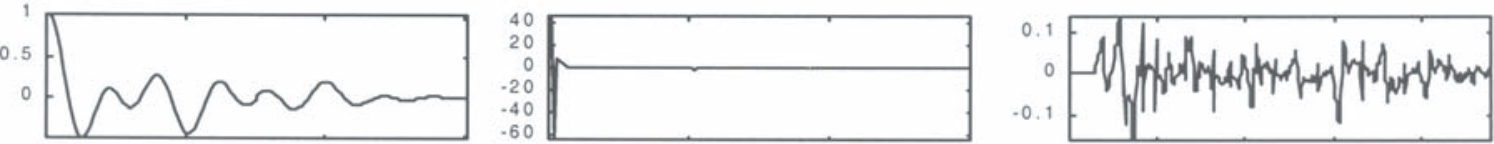

c)
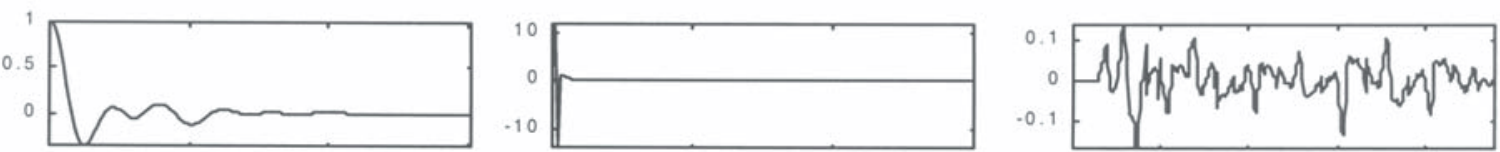

d)
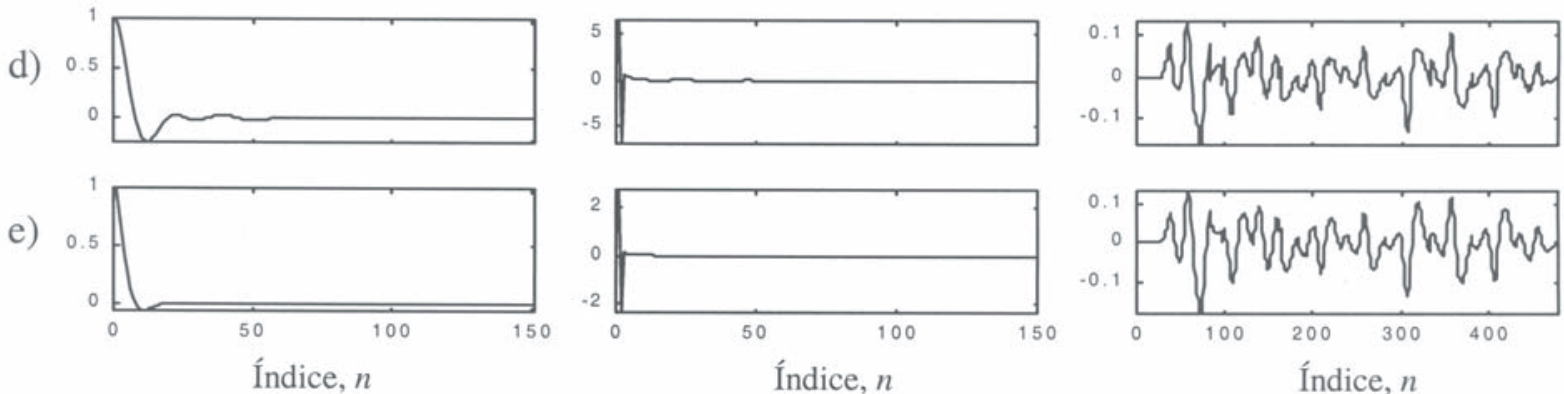

f)

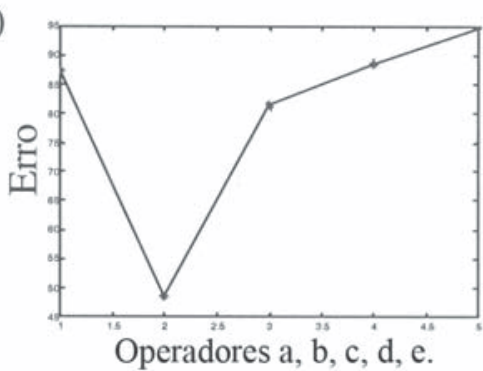

Figura 7 - (Bloco C). Caso fase não-mínima e sem ruído. A coluna esquerda mostra as autocorrelações sob janelas diferentes, a coluna central os operadores longos, $\mathrm{h}_{\mathrm{t}}(\mathrm{n})=\mathrm{h}(\mathrm{n})$, obtidos, e a coluna direita os sismogramas deconvolvidos. a) Janela retangular. b) Janela triangular. c) Janela exponencial com decaimento 30. d) Janela exponencial com decaimento 15. e) Janela exponencial com decaimento 5. f) A parte inferior mostra a variação do erro do operador. Em paralelo, as raízes $\mathbf{z}_{\mathrm{n}}$ dos operadores foram analisadas, e elas estão sistematicamente externas ao círculo unitário e uniformemente distribuídas. A melhor solução é ao redor de b), porém é evidente a degeneração dos resultados com relação à solução da Figura 4.

Figure 7 - (Block C). Case of non-minimum phase without noise. The left column shows the autocorrelations under different windows, the central column shows the long operators, $\mathrm{h}_{\mathrm{L}}(\mathrm{n})=\mathrm{h}(\mathrm{n})$, obtained, and the right column shows the deconvolved seismograms. a) Rectangular window. b) Triangular window. c) Exponential window with decay 30. d) Exponential window with decay 15. e) Exponential window with decay 5 . ff The lower part shows the variation of the operator error. In parallel, the roots $\mathrm{z}_{\mathrm{n}}$ of operators were analyzed, and they are systematically external to the unitary circle, and uniformly distributed. The best solution is around b), but is evident the degeneration of the results with respect to the solution of figure 4. 
a)

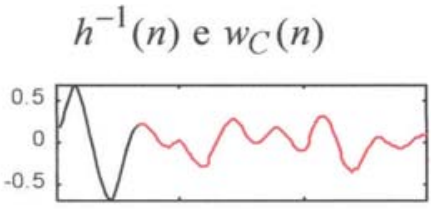

b)

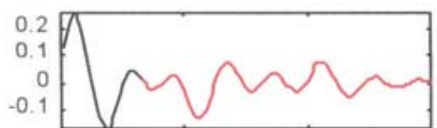

c)

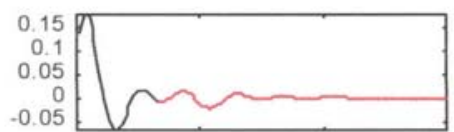

d)

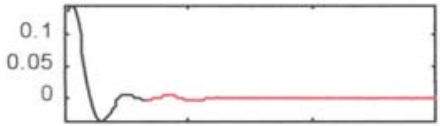

e)

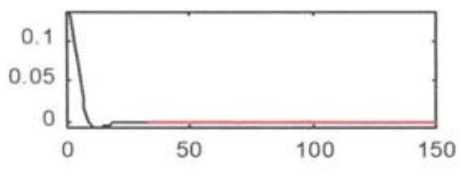

Índice, $n$
$w_{C}^{-1}(n)$
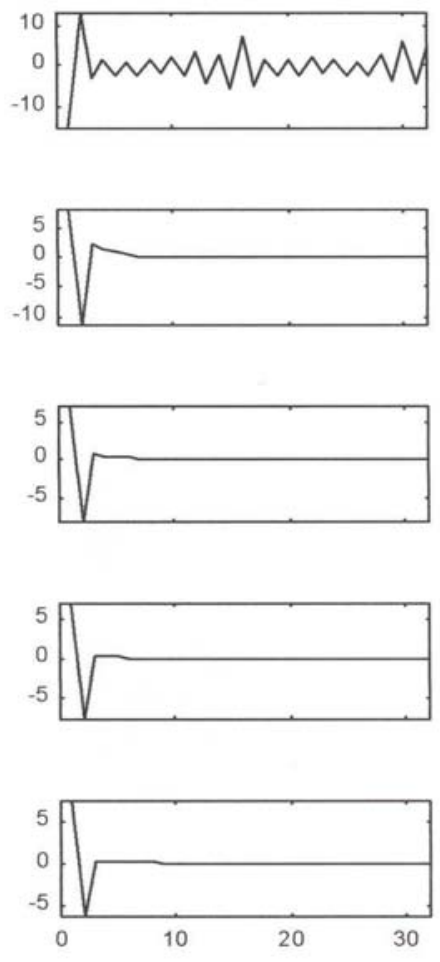

Índice, $n$
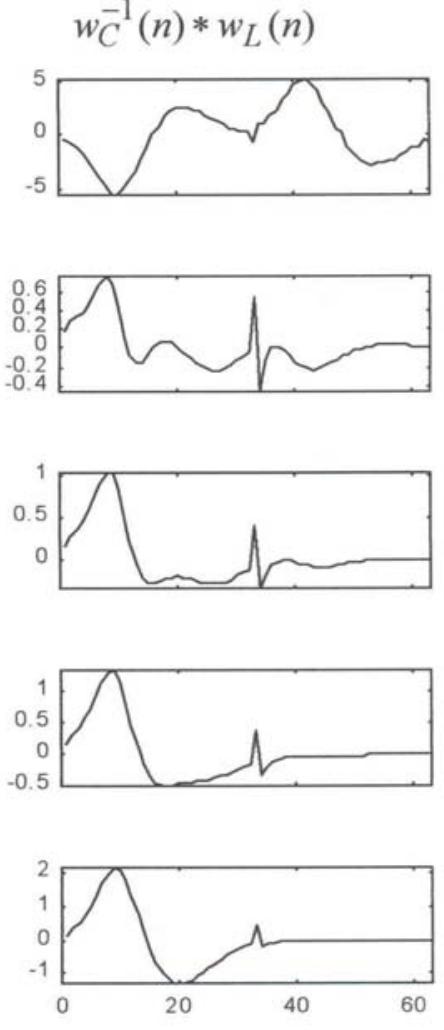

Índice, $n$

Figura 8 - (Bloco C). Caso fase não-mínima e sem ruído. Análise da qualidade dos operadores da Figura 7. A coluna esquerda mostra $h^{-1}(n)=w_{L}(n)$ e $\mathrm{w}_{C}(\mathrm{n})$, a coluna central $\mathrm{w}_{\mathrm{C}}^{-1}(\mathrm{n})$ e a coluna direita $\mathrm{w}_{\mathrm{C}}^{-1}(\mathrm{n}) * \mathrm{w}_{\mathrm{L}}(\mathrm{n})$. As letras a), b), c), d) e e) seguem a mesma sequiência da Figura 7. A melhor solução é ao redor de b) e c).

Figure 8-(Block C). Case of non-minimum phase without noise. Analysis of the quality of the operators shown in Figure 7 . The left column shows $\mathrm{h}^{-1}(\mathrm{n})=\mathrm{w}_{\mathrm{L}}(\mathrm{n})$ and $\mathrm{w}_{\mathrm{C}}(\mathrm{n})$, the central column shows $\mathrm{w}_{\mathrm{C}}^{-1}(\mathrm{n})$, and the right column shows $\mathrm{w}_{\mathrm{C}}^{-1}(\mathrm{n}) * \mathrm{w}_{\mathrm{L}}(\mathrm{n})$. The letters a), b), $\left.\left.\mathrm{c}\right), \mathrm{d}\right)$ and e) follow the same sequence in Figure 7 . The best solution is around b) and c). 
a)

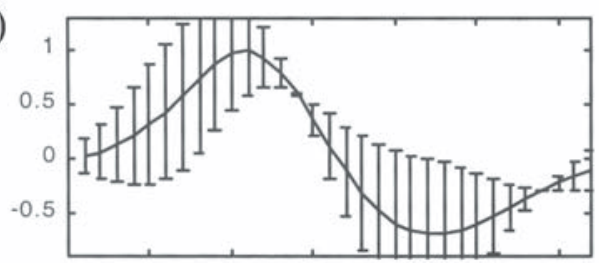

c)

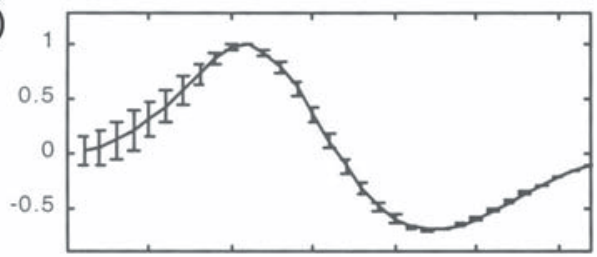

e)

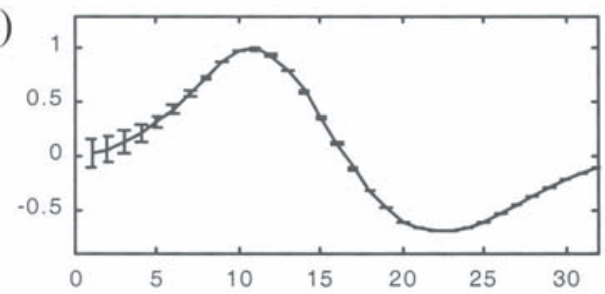

b)

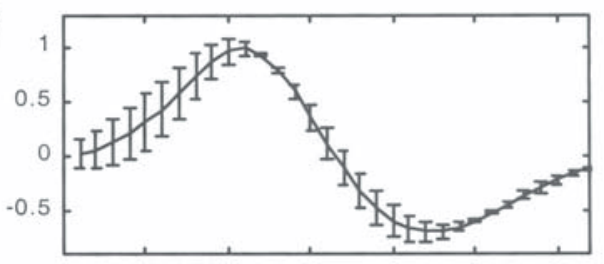

d)

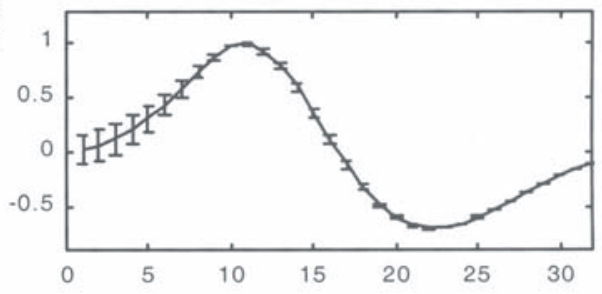

Índice, $n$

Índice, $n$

Figura 9 - (Bloco C). Caso fase não-mínima e sem ruído. Pulsos-fontes efetivos curtos, $\mathrm{w}_{\mathrm{C}}(\mathrm{n})$, recuperados pelos operadores exibidos nas Figuras $7 \mathrm{e} 8$. A linha continua é 0 pulso original, e as barras indicam a diferença para o pulso recuperado. As letras a), b), c), d) e e) seguem a mesma sequiencia das Figuras 7 e 8 .

Figure 9- (Block C). Case of non-minimum phase without noise. Effective short source wavelet, $\mathrm{w}_{\mathrm{C}}(\mathrm{n})$, recovered by the operators shown in Figures 7 and 8. The continuos line is the original wavelet, and the bars indicate the difference from the recovered wavelet. The letters a), b), c), d) and e) follow the same sequence as in Figures 7 and 8. 
a)

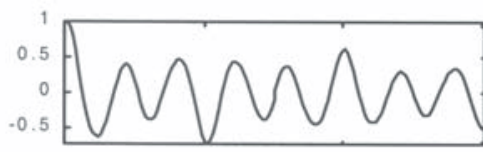

b)

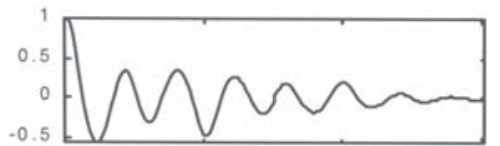

c)

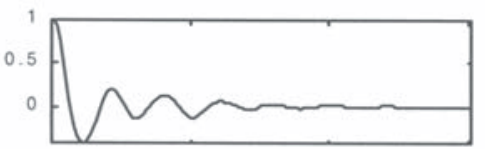

d)

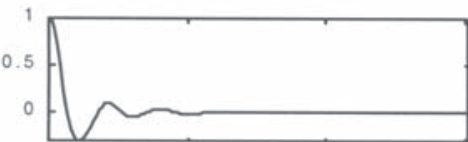

e)

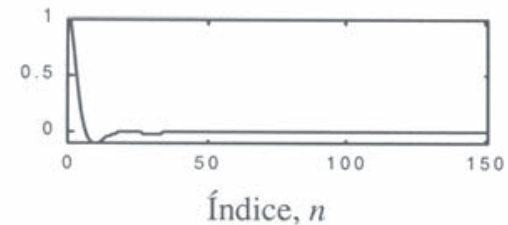

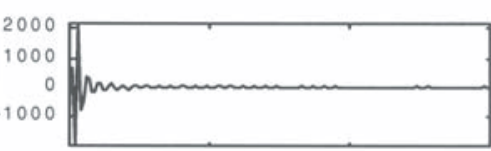
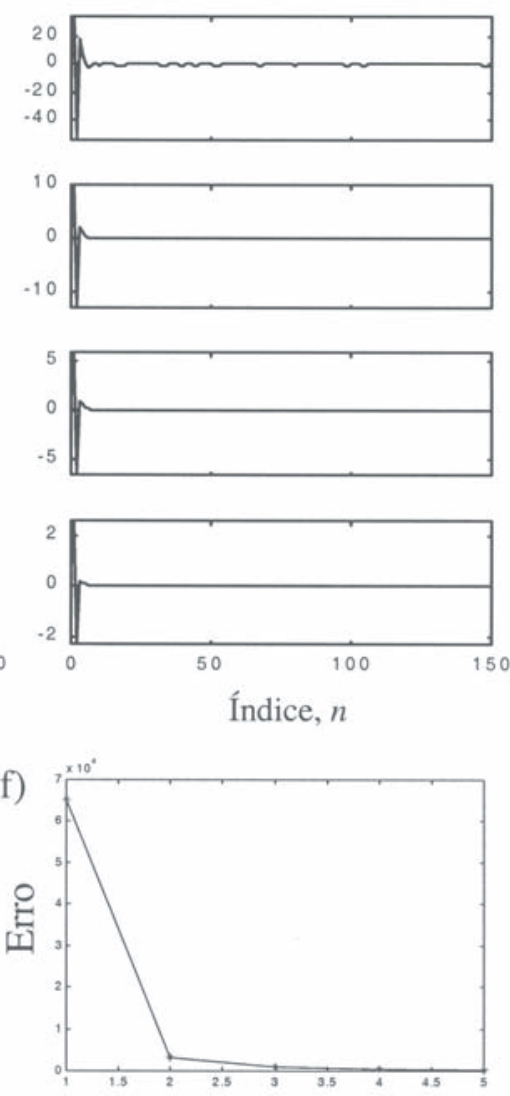

Operadores a, b, c, d, e.
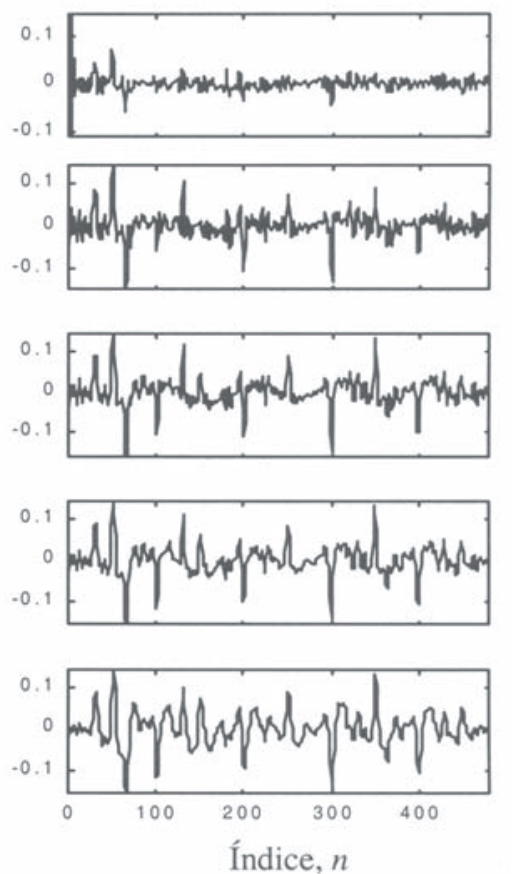

Figura 10 - (Bloco D). Caso fase mínima e com ruído. A coluna esquerda mostra as autocorrelações sob janelas diferentes, a coluna central os operadores longos, $\mathrm{h}_{\mathrm{L}}(\mathrm{n})=\mathrm{h}(\mathrm{n})$ obtidos, e a coluna direita os sismogramas ruidosos deconvolvidos após aplicação do equalizador PBO (40/120) e da descoloração (50\%). a) Janela retangular. b) Janela triangular. c) Janela exponencial com decaimento 30. d) Janela exponencial com decaimento 15. e) Janela exponencial com decaimento 5. f) A parte inferior mostra a variação do erro do operador. Em paralelo, as ráizes $\mathbf{z}_{\mathrm{n}}$ dos operadores foram analisadas, e elas estão sistematicamente externas ao círculo unitário, uniformemente distribuídas e progressivamente mais distanciadas. 0 problema provocado pelo ruído é evidente.

Figure 10 - (Block D). Case of minimum phase with noise. The left column shows the autocorrelation under different windows, the central column shows the long operators, $h_{L}(n)=h(n)$, obtained, and the right column shows the deconvolved noisy seismograms after application of equalizer PBO (40/120) and of whitening (50\%). a) Rectangular window. b) Triangular window. c) Exponential window with decay 30. d) Exponential window with decay 15. e) Exponential window with decay 5. (f) The lower part shows the variation of the operator error. In parallel, the roots $z_{n}$ of the operators were analyzed, and they are systematically external to the unitary circle, uniformly distributed, and progressively distanciated. The problem due to the noise is evident. 


$$
h^{-1}(n) \text { e } w_{C}(n)
$$

a)

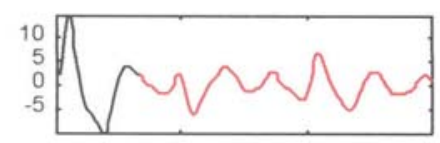

b)

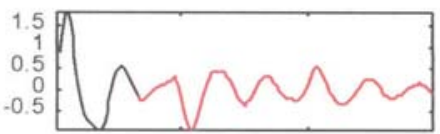

c)

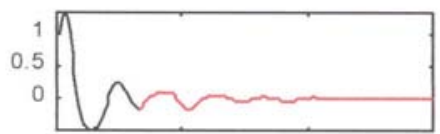

d)

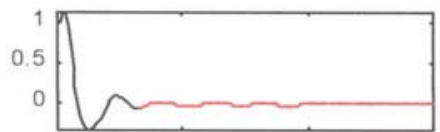

e)

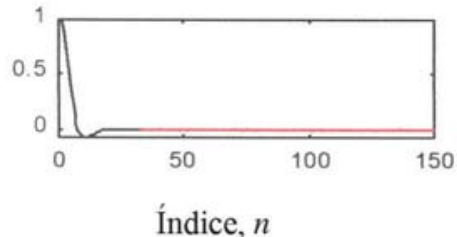

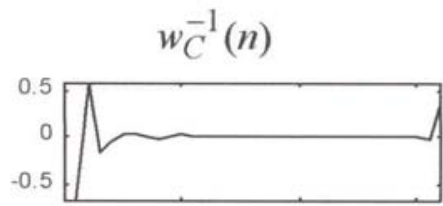
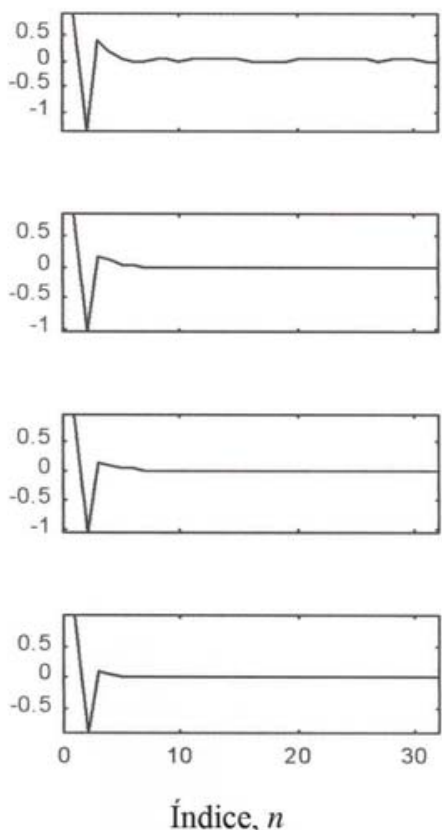
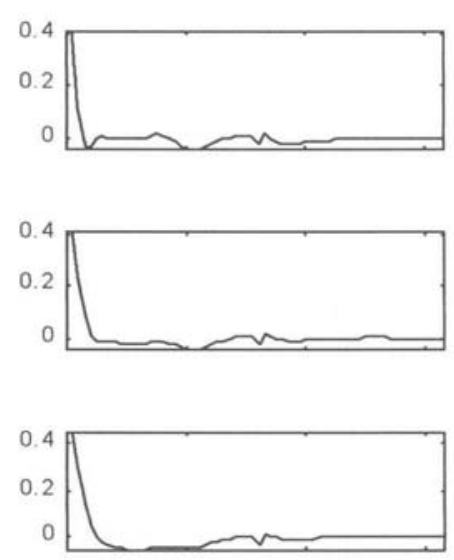

$w_{C}^{-1}(n) * w_{L}(n)$
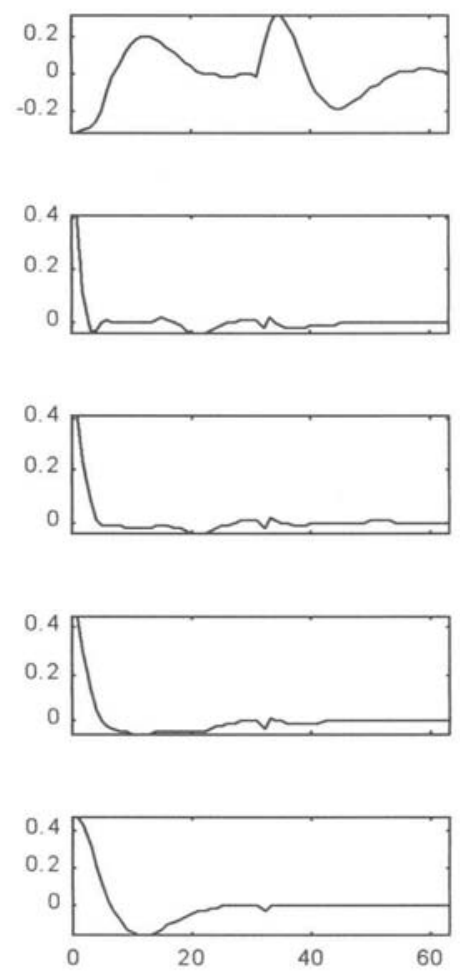

Índice, $n$

Figura 11 - (Bloco D). Caso fase mínima e com rúído. Análise da qualidade dos operadores da Figura 10. A coluna esquerda mostra $\mathrm{h}^{-1}(\mathrm{n})$ e $\mathrm{w}_{\mathrm{C}}(\mathrm{n})$, a coluna central mostra $\mathrm{w}_{\mathrm{C}}^{-1}(\mathrm{n})$, e a coluna direita $\mathrm{w}_{\mathrm{C}}^{-1}(\mathrm{n}) * \mathrm{w}_{\mathrm{L}}(\mathrm{n})$. As letras $\left.\left.\left.\left.\mathrm{a}\right), \mathrm{b}\right), \mathrm{c}\right), \mathrm{d}\right)$ e e) têm a mesma sequênncia da Figura 10. A melhor solução é ao redor de (b) e (c), porém a distorção é evidente.

Figure 71 - (Block D). Case of minimum phase with noise. Analysis of quality of the operators shown in Figure 10. The leff column shows $h^{-1}(n)$ and $w_{C}(n)$, the central column shows $\mathrm{w}_{\mathrm{C}}^{-1}(\mathrm{n})$, and the right column shows $\mathrm{w}_{\mathrm{C}}^{-1}(\mathrm{n}) * \mathrm{w}_{\mathrm{L}}(\mathrm{n})$. The letters a), b), c), d) and e) have the same sequence as in Figure 10. The best solution is around (b) and (c), but the distortion is evident. 
a)

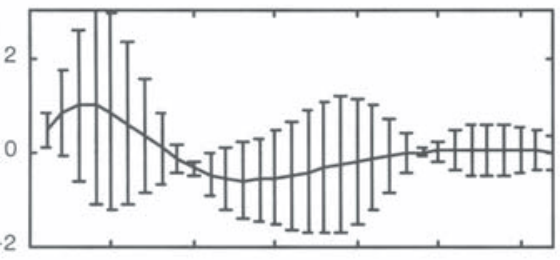

c)

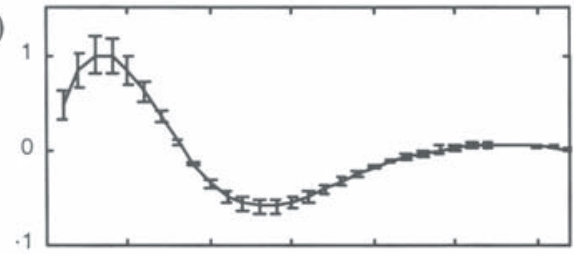

e)

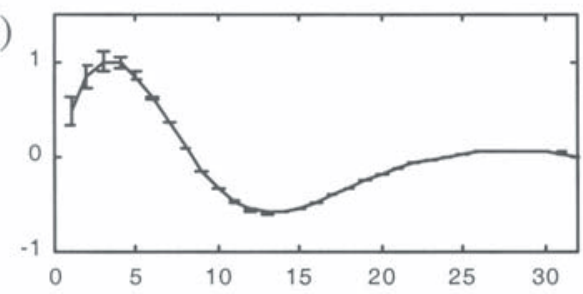

b)

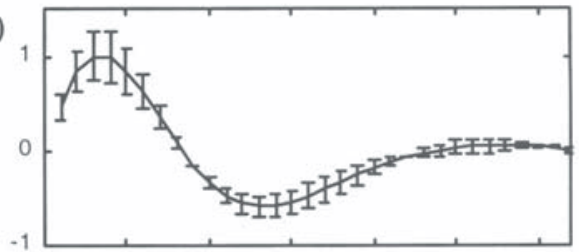

d)

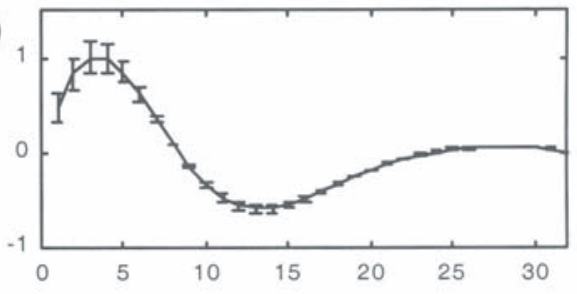

Índice, $n$

Índice, $n$

Figura 12 - (Bloco D). Caso fase mínima e com ruído. Pulsos-fontes efetivos curtos, $\mathrm{w}_{\mathrm{C}}(\mathrm{n})$, recuperados pelos operadores exibidos nas Figuras 10 e 11. A linha continua é o pulso original, e as barras indicam a diferença para o pulso recuperado. As letras a), b), c), d) e e) seguem a mesma sequiência das Figuras 10 e 11.

Figure 12 - (Block D). Case of minimum phase with noise. Effective short source wavelet, $w_{C}(n)$, recovered by the operators shown in Figures 10 and 11. The continuos line is the original wavelet, and the bars indicate the difference from the recovered pulse. The lefters a), b), c), d) and e) follow the same sequence as in Figures 10 and 11. 
Autocorrelações, $\phi_{g g}(n)$

a)

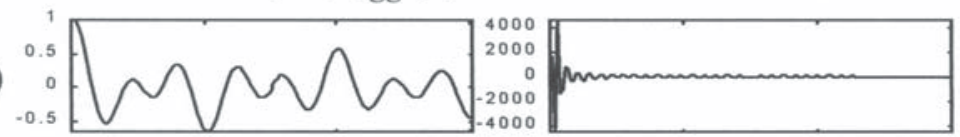

b)

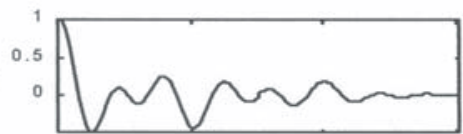

c)

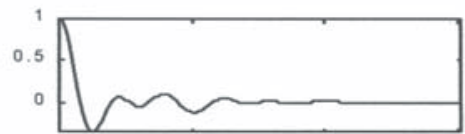

d)

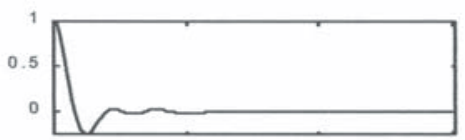

e)

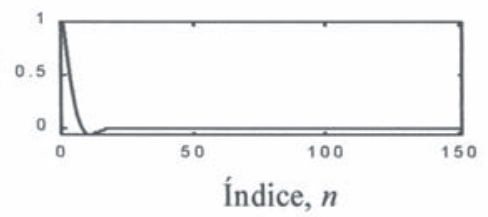

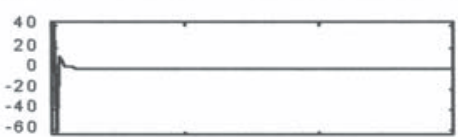
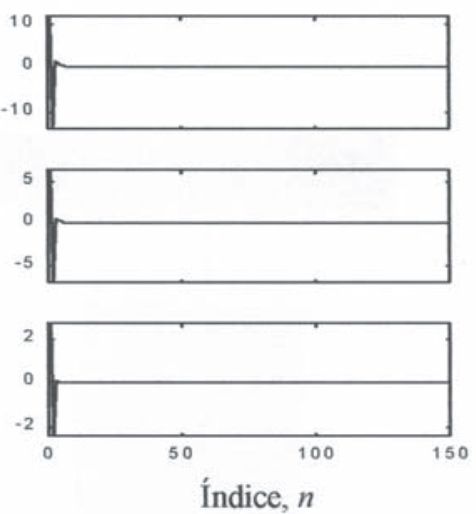

f)

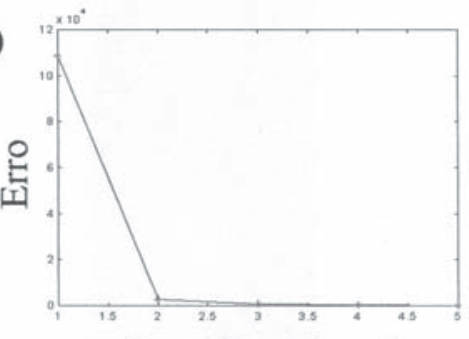

Operadores a, b, c, d, e.
Traços deconvolvidos, $y(n)$
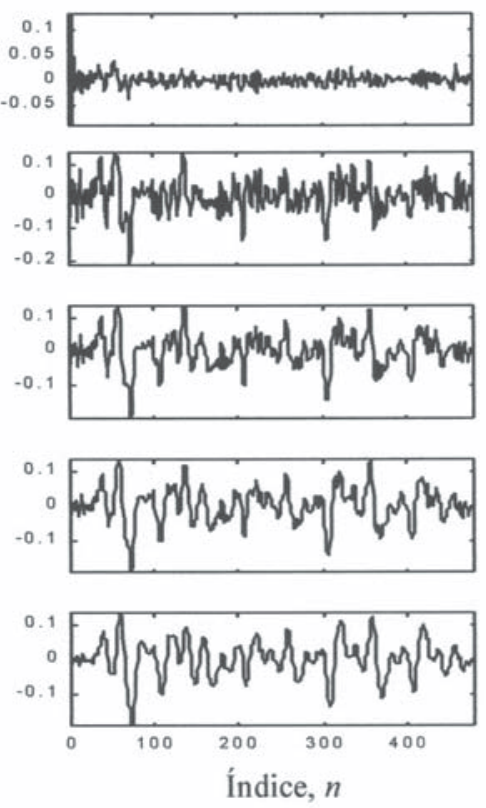

Figura 13 - (Bloco E). Caso fase não-mínima e com ruído. A coluna esquerda mostra autocorrelações sob janelas diferentes, a coluna central mostra os operadores longos, h(n), obtidos, e na coluna direita estão os sismogramas com ruído deconvolvidos após aplicaçãa do equalizador (PB0, 40/120) e da descoloração (50\%). a) Janela retangular. b) Janela triangular. c) Janela exponencial com decaimento 30. d) Janela exponencial com decaimento 15. e) Janela exponencial com decaimento 5. f) A parte inferior mostra a variação do erro do operador. Em paralelo, as raízes $\mathbf{z}_{\mathrm{n}}$ dos operadores foram analisadas, e elas estãa externas ao círculo unitário e uniformemente distribuídas. É evidente a degeneração provocada pelo ruído.

Figure 13 - (Block E). Case of non-minimum phase with noise. The left column shows the autocorrelations under different windows, the central column shows the long operators, $h(n)$, obtained, and in the right column shows the deconvolved seismograms with noise after application of equalizer (PBO, 40/120) and of whitening (50\%). a) Rectangular window. b) Triangular window. c) Exponential window with decay 30. d) Exponential window with decay 15. e) Exponential window with decay 5. f) The lower part shows the variation of the error of the operator. In parallel, the roots $z_{n}$ of the operators were analyzed, they are external to the unitary circle and uniformly distributed. It is evident the degeneration caused by the noise. 
a)

$$
h^{-1}(n) \text { e } w_{C}(n)
$$
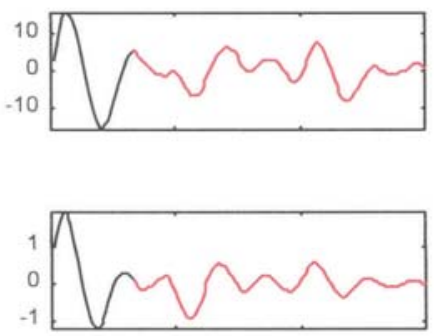

b)

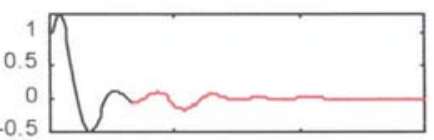

d)

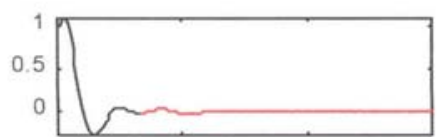

e)

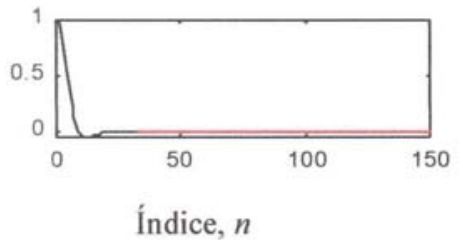

$w_{C}^{-1}(n)$
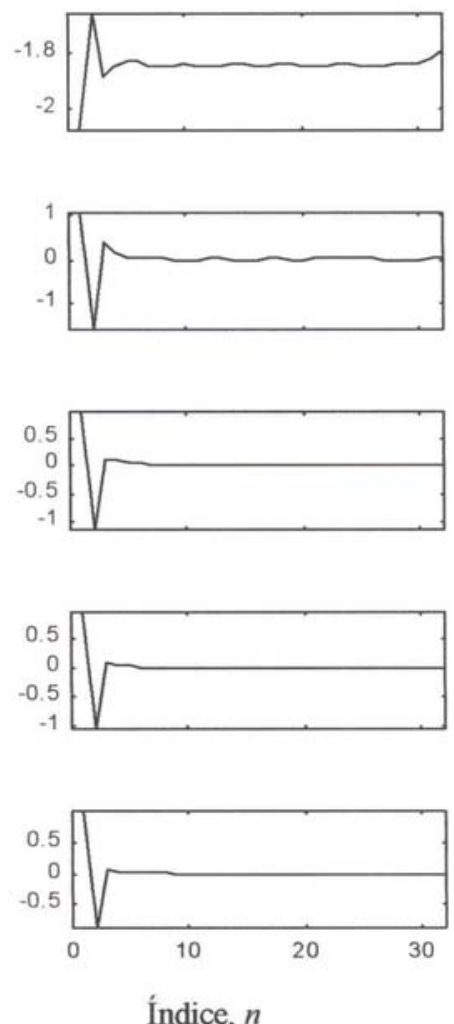

Índice, $n$

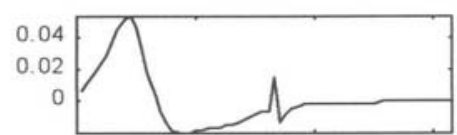

$$
w_{C}^{-1}(n) * w(n)
$$
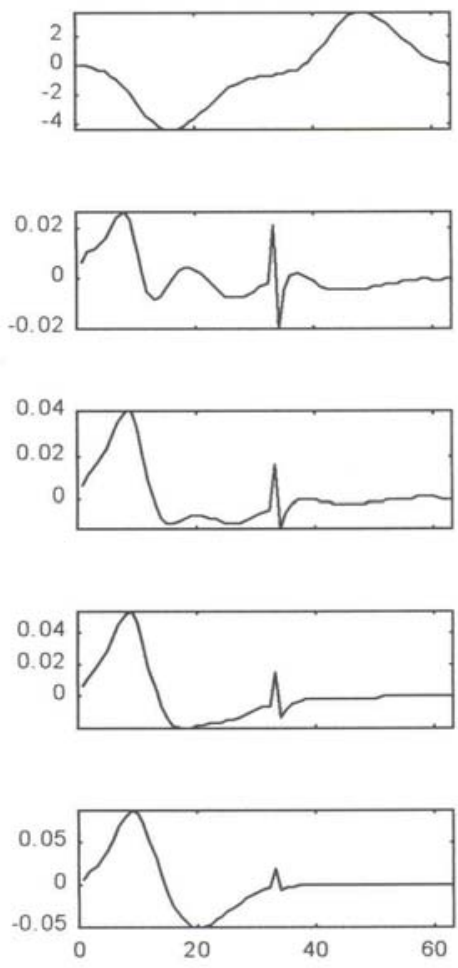

Índice, $n$

Figura 14 - (Bloco E). Caso fase não-mínima e com rú́do. Análise da qualidade dos operadores da Figura 13. A coluna esquerda mostra $\mathrm{h}^{-1}(\mathrm{n})=\mathrm{w}_{\mathrm{L}}(\mathrm{n})$ e $\mathrm{w}_{C}(\mathrm{n})$, a coluna central $\mathrm{w}_{\mathrm{C}}^{-1}(\mathrm{n})$ e a coluna direita $\mathrm{w}_{\mathrm{C}}^{-1}(\mathrm{n}) * \mathrm{w}_{\mathrm{L}}(\mathrm{n})$. As letras a), b), c), d) e e) seguem a mesma sequênncia da Figura 13. Não é claro como selecionar uma solução a partir destes resultados.

Figure 14 - (Block E). Case of non-minimum phase with noise. Analysis of the quality of the operators shown in Figure 13. The left column shows $h^{-1}(n)=w_{L}(n)$ and $w_{C}(n)$, the central column shows $\mathrm{w}_{\mathrm{C}}^{-1}(\mathrm{n})$, and the right column shows $\mathrm{w}_{\mathrm{C}}^{-1}(\mathrm{n}) * \mathrm{w}_{\mathrm{L}}(\mathrm{n})$. The letters $\left.\left.\left.\mathrm{a}\right), \mathrm{b}\right), \mathrm{c}\right)$, d) and e) follow the same sequence of Figure 13 . It is not clear how to select a solution starting from these results. 
a)

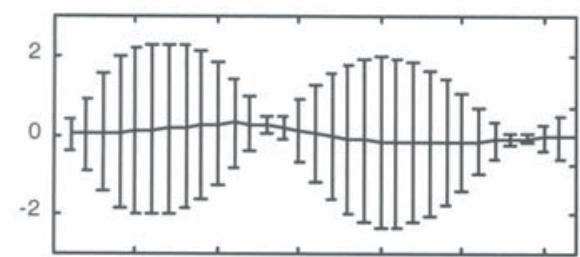

c)

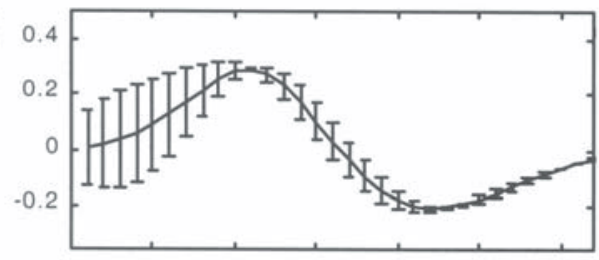

e)

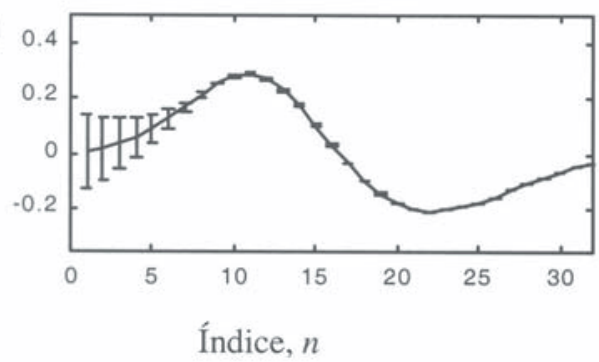

b)

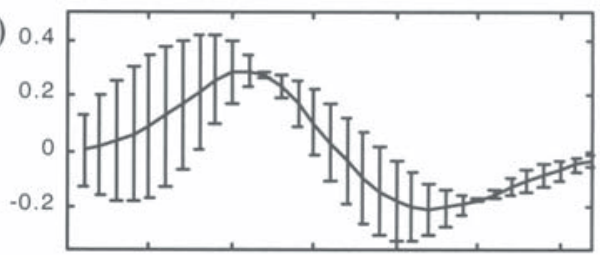

d)

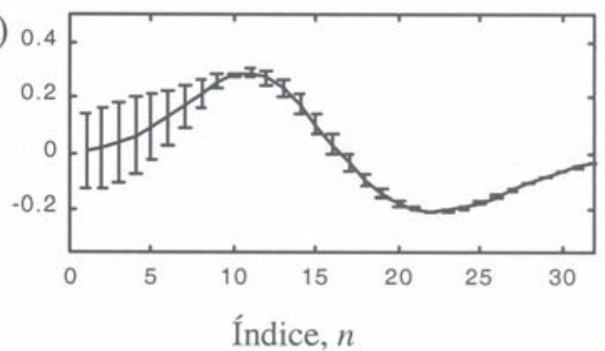

Figura 15 - (Bloco E). Caso fase não-mínima e com ruído. Pulsos-fontes efetivos curtos, $\mathrm{w}_{\mathrm{C}}(\mathrm{n})$, recuperados pelos operadores exibidos nas Figuras 13 e 14. A linha continua é o pulso original, e as barras indicam a diferença para o pulso recuperado. As letras a), b), c), d) e e) seguem a mesma sequiência das Figuras 13 e 14.

Figure 15 - (Block E). Case of non-minimum phase with noise. Effective short source wavelet, $\mathrm{w}_{\mathrm{C}}(\mathrm{n})$, recovered by the operators shown in Figures 13 and 14 . The continuos line is the original wavelet, and the bars indicate the difference to the recovered wavelet. The (etters a), b), c), d) and e) follow the same sequence as in Figures 13 and 14. 
Autocorrelações, $\phi_{g g}(n)$

a)

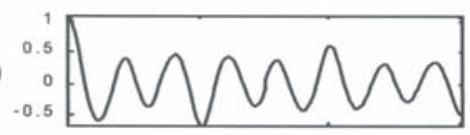

b)

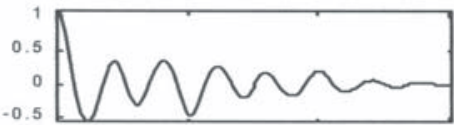

c)

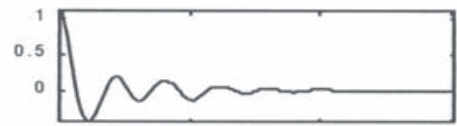

d)

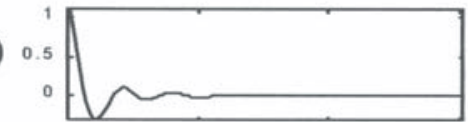

e)

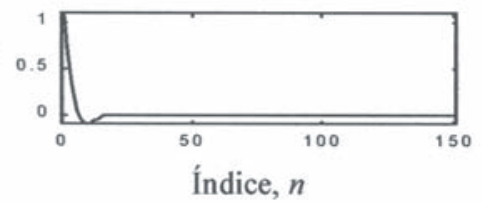

Operadores, $h(n)$
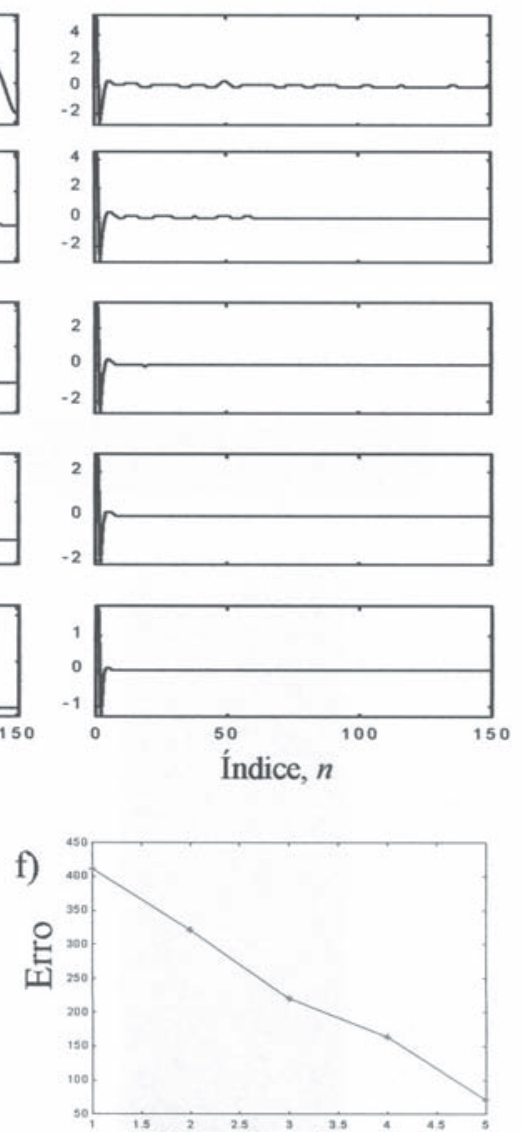

Operador a, b, c, d, e.
Traços deconvolvidos, $y(n)$
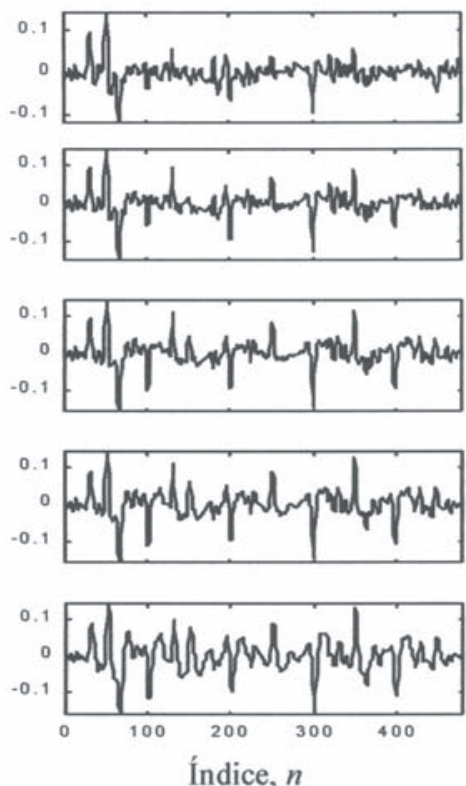

Figura 16 - (Bloco F). Efeito do equalizador (PBO, 40/120) e da descoloração (10\%) para o caso fase mínima, com ruído e traço com ganho dinâmico. A coluna esquerda mostra as autocorrelações sob janelas diferentes, a coluna central mostra os operadores longos, $h_{L}(n)=h(n)$, obtidos, e a coluna direita os sismogramas deconvolvidos após aplicação do equalizador e da descoloração. a) Janela retangular. b) Janela triangular. c) Janela exponencial com decaimento 30. d) Janela exponencial com decaimento 15. e) Janela exponencial com decaimento 5. f) A parte inferior mostra a variação do erro do operador. Em paralelo, as raízes $\mathrm{z}_{\mathrm{n}}$ dos operadores foram analisadas, e elas estão sistematicamente externas ao círculo unitário e uniformemente distribuídas.

Figure 16 - (Block F). Effects of equalizer (PBO, 40/120) and of whitening (10\%) for the case of minimum phase with noise and dynamic gain. The left column shows the autocorrelation under different windows, the central column shows the long operators, $\mathrm{h}_{\mathrm{L}}(\mathrm{n})=\mathrm{h}(\mathrm{n})$, obtained, and the right column shows the deconvolved seismograms after application of equalizer and of whitening. a) Rectangular window. b) Triangular window. c) Exponential window with decay 30. d) Exponential window with decay 15. e) Exponential window with decay 5. f) The lower part shows the variation of the error of the operator. In parallel, the $\mathrm{z}_{\mathrm{n}}$ roots of the operators were analyzed, they are systematically external to the unitary circle and uniformly distributed. 
Autocorrelações, $\phi_{g g}(n)$

a)

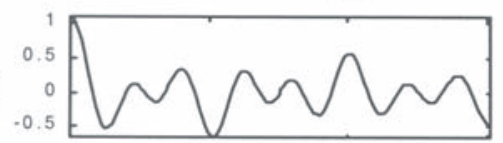

b)

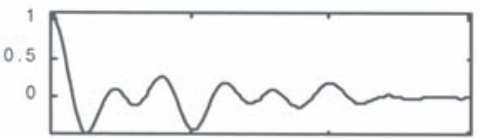

c)

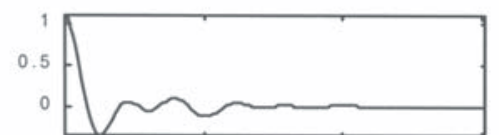

d)

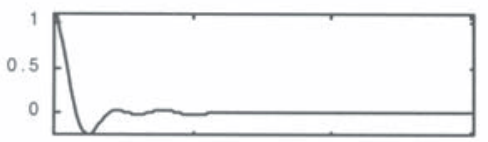

e)

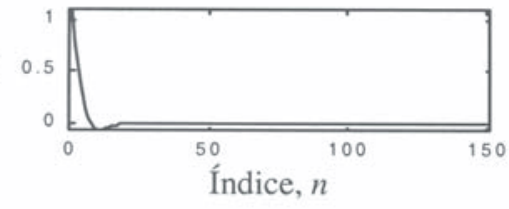

Operadores, $h(n)$
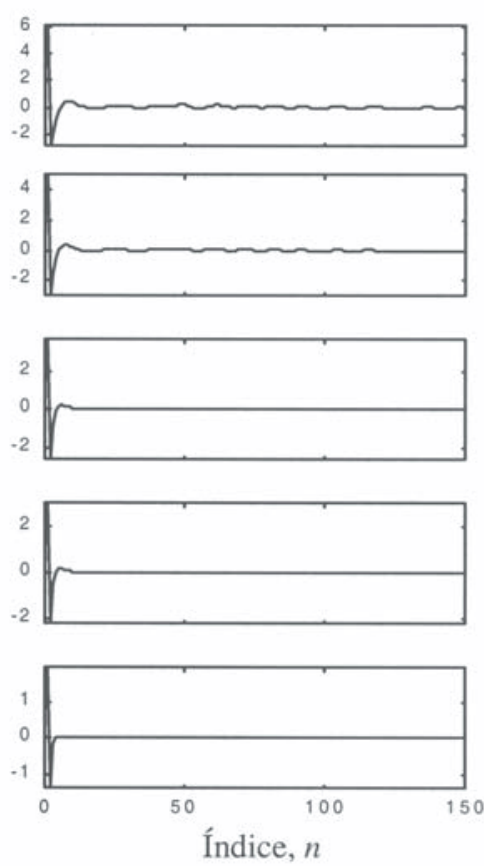

f)

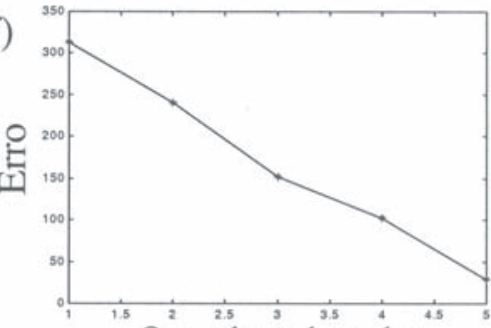

Operador a, b, c, d, e.
Traços deconvolvidos, $y(n)$
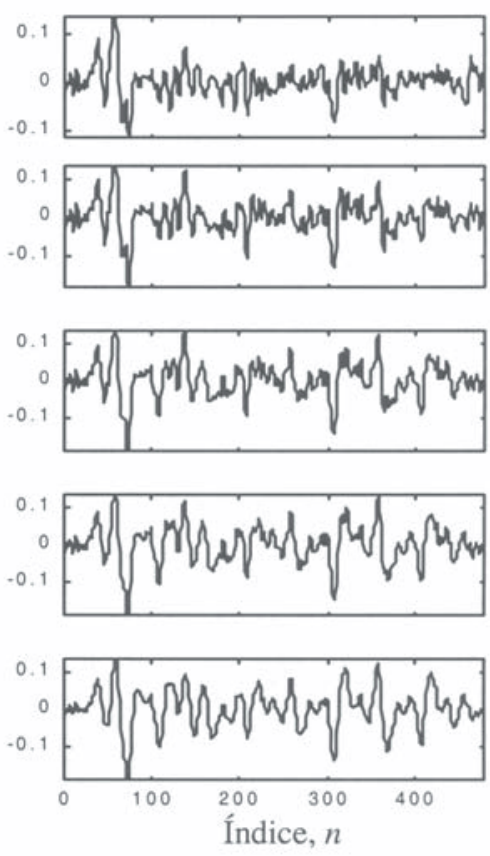

Figura 17 - (Bloco F). Efeito do equalizador (PBO, 40/120) e da descoloração (10\%) para 0 caso fase não-mínima, com ruído e traço com ganho dinâmico. A coluna esquerda mostra as autocorrelações sob janelas diferentes, a coluna central mostra os operadores longos, $h_{L}(n)=h(n)$, obtidos, e a coluna direita mostra os sismogramas deconvolvidos. a) Janela retangular. b) Janela triangular. c) Janela exponencial com decaimento 30. d) Janela exponencial com decaimento 15. e) Janela exponencial com decaimento 5. A parte inferior mostra a variação do erro do operador. Em paralelo, as rázes $\mathrm{z}_{\mathrm{n}}$ dos operadores foram analisadas, e elas estão sistematicamente externas ao círculo unitário e uniformemente distribuídas.

Figure 17 - (Block F). Effects of equalizer (PBO, 40/120) and of whitening (10\%) for the case of non-minimum phase with noise and with dynamic gain. The left column shows the autocorrelations under different windows, the central column shows the long operators, $\mathrm{h}_{\mathrm{L}}(\mathrm{n})=\mathrm{h}(\mathrm{n})$, obtained, and the right column shows the deconvolved seismograms. a) Rectangular window. b) Triangular window. c) Exponential window with decay 30. d) Exponential window with decay 15. e) Exponential window with decay 5. The lower part shows the variation of the error of the operator. In parallel, the roots $\mathrm{z}_{\mathrm{n}}$ of the operators were analyzed, and they are systematically external to the unitary circle and uniformly distributed. 


\section{NOTAS SOBRE OS AUTORES}

Lourenildo Williame Barbosa Leite é bacharel em Geologia pela Universidade Federal de Pernambuco (1968). Mestrado (1974) e Doutorado (1983) pela Saint Louis University, Missouri, USA. Professor do Departamento de Geofísica da Universidade Federal do Pará a partir de 1979. Coordenador do Curso de Pós Graduação em Geofísica, CG/UFPa. Orientador de teses de doutorado e dissertações de mestrado. Leciona disciplinas voltadas à sísmica, sismologia, tratamento e inversão de dados geofísicos. Membro da SEG, SSA, SBGf, SBMAC e SBG.

Fábio José da Costa Alves é Licenciado Pleno em Matemática pela UNESPa (1989). Graduado em Engenharia Civil pela Universidade Federal do Pará (1994). Mestre em Geofísica pelo Curso de Pós-Graduação em Geofísica da UFPa (1999). Cursando o Doutorado em Geofísica da UFPa (2001). Membro da SBGf e SBMAC. 
